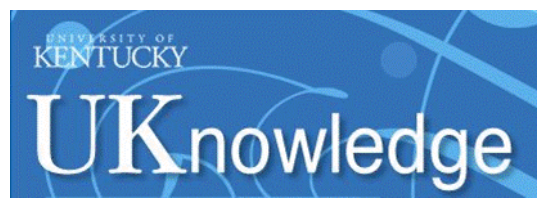

University of Kentucky

UKnowledge

$1-1-2015$

\title{
Lattice Study of Quark and Glue Momenta and Angular Momenta in the Nucleon
}

\author{
M. Deka \\ Bogoliubov Laboratory of Theoretical Physics, Russia \\ T. Doi \\ RIKEN, Japan \\ Y. B. Yang \\ Chinese Academy of Sciences, China \\ B. Chakraborty \\ University of Glasgow, UK
}

S. J. Dong

University of Kentucky

See next page for additional authors

Follow this and additional works at: https://uknowledge.uky.edu/physastron_facpub

Part of the Astrophysics and Astronomy Commons, and the Physics Commons

Right click to open a feedback form in a new tab to let us know how this document benefits you.

\section{Repository Citation}

Deka, M.; Doi, T.; Yang, Y. B.; Chakraborty, B.; Dong, S. J.; Draper, Terrence; Glatzmaier, M.; Gong, M.; Lin, H. W.; Liu, Keh-Fei; Mankame, D.; Mathur, N.; and Streuer, T., "Lattice Study of Quark and Glue Momenta and Angular Momenta in the Nucleon" (2015). Physics and Astronomy Faculty Publications. 309.

https://uknowledge.uky.edu/physastron_facpub/309

This Article is brought to you for free and open access by the Physics and Astronomy at UKnowledge. It has been accepted for inclusion in Physics and Astronomy Faculty Publications by an authorized administrator of UKnowledge. For more information, please contact UKnowledge@lsv.uky.edu. 


\section{Lattice Study of Quark and Glue Momenta and Angular Momenta in the Nucleon}

Digital Object Identifier (DOI)

http://dx.doi.org/10.1103/PhysRevD.91.014505

\section{Notes/Citation Information}

Published in Physical Review D: Particles, Fields, Gravitation, and Cosmology, v. 91, no. 1, article 014505, p. 1-21.

(C2015 American Physical Society

The copyright holder has granted permission for posting the article here.

This work is a result of the $\mathrm{XQCD}$ Collaboration.

\section{Authors}

M. Deka, T. Doi, Y. B. Yang, B. Chakraborty, S. J. Dong, Terrence Draper, M. Glatzmaier, M. Gong, H. W. Lin, Keh-Fei Liu, D. Mankame, N. Mathur, and T. Streuer 
PHYSICAL REVIEW D 91, 014505 (2015)

\title{
Lattice study of quark and glue momenta and angular momenta in the nucleon
}

\author{
M. Deka, ${ }^{1, *}$ T. Doi, ${ }^{2}$ Y. B. Yang, ${ }^{3, \dagger}$ B. Chakraborty, ${ }^{4}$ S. J. Dong, ${ }^{5}$ T. Draper, ${ }^{5}$ M. Glatzmaier, ${ }^{5}$ M. Gong, ${ }^{5}$ \\ H. W. Lin, ${ }^{6}$ K. F. Liu, ${ }^{5, \$}$ D. Mankame,${ }^{5}$ N. Mathur, ${ }^{7}$ and T. Streuer ${ }^{8}$ \\ $(\chi \mathrm{QCD}$ Collaboration)
}

\author{
${ }^{1}$ Bogoliubov Laboratory of Theoretical Physics, JINR, 141980 Dubna, Russia \\ ${ }^{2}$ Theoretical Research Division, Nishina Center, RIKEN, Wako 351-0198, Japan \\ ${ }^{3}$ Institute of High Energy Physics, Chinese Academy of Sciences, Beijing 1000190, China \\ ${ }^{4}$ School of Physics and Astronomy, University of Glasgow, Glasgow G12 8QQ, United Kingdom \\ ${ }^{5}$ Department of Physics and Astronomy, University of Kentucky, Lexington, Kentucky 40506, USA \\ ${ }^{6}$ Department of Physics, University of Washington, Seattle, Washington 98195, USA \\ ${ }^{7}$ Department of Theoretical Physics, Tata Institute of Fundamental Research, Mumbai 40005, India \\ ${ }^{8}$ Institute for Theoretical Physics, University of Regensburg, 93040 Regensburg, Germany
}

(Received 29 March 2014; published 16 January 2015)

\begin{abstract}
We report a complete calculation of the quark and glue momenta and angular momenta in the proton. These include the quark contributions from both the connected and disconnected insertions. The quark disconnected insertion loops are computed with $Z_{4}$ noise, and the signal-to-noise ratio is improved with unbiased subtractions. The glue operator is comprised of gauge-field tensors constructed from the overlap operator. The calculation is carried out on a $16^{3} \times 24$ quenched lattice at $\beta=6.0$ for Wilson fermions with $\kappa=0.154,0.155$, and 0.1555 , which correspond to pion masses at 650,538 , and $478 \mathrm{MeV}$, respectively. The chirally extrapolated $u$ and $d$ quark momentum/angular momentum fraction is found to be $0.64(5) / 0.70(5)$, the strange momentum/ angular momentum fraction is $0.024(6) / 0.023(7)$, and that of the glue is $0.33(6) / 0.28(8)$. The previous study of quark spin on the same lattice revealed that it carries a fraction of 0.25(12) of proton spin. The orbital angular momenta of the quarks are then obtained from subtracting the spin from their corresponding angular momentum components. We find that the quark orbital angular momentum constitutes $0.47(13)$ of the proton spin with almost all of it coming from the disconnected insertions.
\end{abstract}

DOI: 10.1103/PhysRevD.91.014505

PACS numbers: 12.38.Gc, 11.15.Ha, 11.30.Rd, 14.20.Dh

\section{INTRODUCTION}

Determining the contributions from quarks and gluons to the nucleon spin is one of the most challenging issues in QCD both experimentally and theoretically. Since the contribution from the quark spin is found out to be small ( $\sim 25 \%$ of the total proton spin) from the global analysis of deep inelastic scattering data [1], it is expected that the rest should come from glue spin and the orbital angular momenta of quarks and glue.

The quark spin contribution from $u, d$, and $s$ has been studied on the lattice [2,3] since 1995 using either the quenched approximation or dynamical fermions with heavier quark mass [4]. Recently, it has been carried out with light dynamical fermions $[5,6]$ and only for strange quarks (not renormalized) in Ref. [7]. The calculation of disconnected insertion (DI) contributions to quark spin

\footnotetext{
*mpdeka@theor.jinr.ru ybyang@pa.uky.edu *liu@pa.uky.edu
}

from $u, d, s$, and $c$ using the anomalous Ward identity with light overlap fermions is under progress [8].

As for the quark orbital angular momenta, lattice calculations have been carried out for the connected insertions (CI) [9-15]. They are obtained by subtracting the quark spin contributions from those of the quark angular momenta. It has been shown that the contributions from $u$ and $d$ quarks mostly cancel each other. Thus, for connected insertion, quark orbital angular momenta turn out to be small in the quenched calculation $[9,10]$ and nearly zero in dynamical fermion calculations [11-15]. On the other hand, gluon helicity distribution $\Delta G(x) / G(x)$ from COMPASS, STAR, HERMES, and PHENIX experiments is found to be close to zero [16-20]. The latest global fit [21] with the inclusion of the polarized deep inelastic scattering (DIS) data from COMPASS [22] and the 2009 data from the Relativistic Heavy Ion Collider (RHIC) [21] gives a glue contribution $\int_{0.05}^{0.2} \Delta g(x) d x=0.1 \pm_{0.07}^{0.06}$ to the total proton spin of $1 / 2 \hbar$ with a sizable uncertainty. Furthermore, it is argued based on analysis of single-spin asymmetry in unpolarized lepton scattering from a transversely polarized nucleon that the glue 
orbital angular momentum is absent [23]. In this given context, we know from lattice and experiments thus far that $\sim 25 \%$ of the proton spin comes from the quark spin, CI orbital angular momenta have negligible contributions, and gluon helicity from experiments is $\sim 20 \%$. Since we have not been able to identify the rest $(\sim 50 \%)$ of the proton spin, it appears that we have encountered a "dark spin" conundrum.

In this work, we give a complete calculation of the quark and glue momenta and angular momenta on a quenched lattice. The quark contributions to both the connected and disconnected insertions are included. We have been able to obtain the glue momentum and angular momentum for the first time, mainly because the overlap operator is used for the gauge-field tensor [24,25], the construction of which is much less noisy than that from thin gauge links. Combining with earlier work on the quark spin [2], we obtain the quark orbital angular momenta. We find that the $u$ and $d$ quark orbital angular momenta indeed largely cancel in the connected insertion. However, their contributions together with that of the strange quark are large ( $\sim 50 \%)$ in the DI where the quark spin for the $u, d$, and $s$ in DI are large and negative.

These results from our lattice calculations are improved by satisfying the momentum and angular momentum sum rules for the quark and glue contributions. The renormalization and mixing of the quark and glue energy-momentum operators are performed perturbatively, and the final results are reported in the $\overline{\mathrm{MS}}$ scheme at $2 \mathrm{GeV}$.

The manuscript is organized as follows. Section II discusses the general formalism about the quark and glue energy-momentum tensor operators and their contributions to the proton momenta and angular momenta via the associated form factors. The lattice formulation is presented in Sec. III. In Sec. IV, the stochastic method for computing the quark loops in the disconnected insertions and the computation of glue operators constructed from the overlap operator are described. Utilization of unbiased subtraction and discrete symmetries for variance reduction is also discussed in the same section. The choice of momenta and the separation of the $T_{1}, T_{2}$, and $T_{3}$ form factors are described in Secs. V and VI, respectively. The renormalization of the quark and glue energy-momentum tensor operators and their mixing and matching to the $\overline{\mathrm{MS}}$ scheme at the $2 \mathrm{GeV}$ scale is discussed in Sec. VIII D. We give the numerical details in Sec. VII and the results in Sec. VIII. We conclude with a summary in Sec. IX.

\section{GENERAL FORMALISM}

The Lorentz group generators, $J^{\mu \nu}$, for angular momentum operators are given by [26]

$$
J^{\mu \nu} \equiv \int d^{3} x M^{0 \mu \nu}(\vec{x}) .
$$

Here, $M^{0 \mu \nu}$ is the angular momentum density, which is defined as

$$
M^{\mu \nu \alpha}(x)=\mathcal{T}^{\{\mu \alpha\}} x^{\nu}-\mathcal{T}^{\{\mu \nu\}} x^{\alpha},
$$

where $\mathcal{T}^{\{\mu \nu\}}$ is the energy-momentum tensor and has the Belinfante-improved form. It is gauge invariant and conserved [27], and $\{\cdots\}$ stands for the symmetrization of indices.

One can construct gauge-invariant energy-momentum tensor operators for quarks and gluons separately. As a result, we can write $\mathcal{T}^{\{\mu \nu\}}$ as the gauge-invariant sum

$$
\mathcal{T}^{\{\mu \nu\}}=\mathcal{T}^{\{\mu \nu\} q}+\mathcal{T}^{\{\mu \nu\} g},
$$

where the superscripts, $q$ and $g$, stand for quarks and gluons, respectively. The operators, $\mathcal{T}\{0 i\} q$ and $\mathcal{T}^{\{0 i\} g}$, have the following twist-2 forms:

$$
\mathcal{T}^{\{0 i\} q}=\frac{i}{4} \sum_{f} \bar{\psi}_{f}\left[\gamma^{0} \overrightarrow{D^{i}}+\gamma^{i} \overrightarrow{D^{0}}-\gamma^{0} \stackrel{\leftarrow}{D^{i}}-\gamma^{i} \overleftarrow{D^{0}}\right] \psi_{f},
$$

and

$$
\begin{aligned}
\mathcal{T}^{\{0 i\} g} & =-\frac{1}{2} \sum_{k=1}^{3}\left[G^{a, 0 k} G_{k}^{a, i}+G^{a, i k} G_{k}^{a, 0}\right] \\
& =-\frac{1}{2} \sum_{k=1}^{3} 2 \operatorname{Tr}^{\text {color }}\left[G^{0 k} G_{k}^{i}+G^{i k} G_{k}^{0}\right] .
\end{aligned}
$$

In Eq. (4), $\psi_{f}$ denotes the quark field operator for the flavor, $f$. In Eq. (5), $a$ is the color index, and $G$ 's are the gaugefield strength tensors.

Using Eqs. (1), (2), and (3), one can write the $i$ th component of the angular momentum operators for quarks and gluons, $\vec{J}^{q, g}$, as

$$
J_{i}^{q, g}=\frac{1}{2} \epsilon^{i j k} \int d^{3} x\left(\mathcal{T}^{\{0 k\} q, g} x^{j}-\mathcal{T}^{\{0 j\} q, g} x^{k}\right)
$$

so that the total angular momentum is

$$
\vec{J}=\vec{J}^{q}+\vec{J}^{g}
$$

In a similar manner, the linear momentum operators are given by

$$
P_{i}^{q, g}=\int d^{3} x \mathcal{T}^{\{0 i\} q, g} .
$$

Substituting the explicit form of $\mathcal{T}^{\{0 i\} q}$ from Eq. (4) into Eq. (6), and using the QCD equations of motion, one can obtain the gauge-invariant decomposition of $\vec{J}^{q}$ as $[28,29]$ 


$$
\vec{J}^{q}=\int d^{3} x\left[\frac{1}{2} \bar{\psi} \vec{\gamma} \gamma^{5} \psi+\psi^{\dagger}\{\vec{x} \times(i \vec{D})\} \psi\right],
$$

where the color indices are suppressed. From the spin- $\frac{1}{2}$ field theory, one can identify the first term of Eq. (9) as the quark spin operator $\left(\frac{1}{2} \vec{\Sigma}^{q}\right)$ and the second term as the orbital angular momentum operator $\left(\vec{L}^{q}\right)$. Thus, we can write the total angular momentum for quarks as

$$
\vec{J}^{q}=\frac{1}{2} \vec{\Sigma}^{q}+\vec{L}^{q} .
$$

Similarly, using equations of motion and superpotentials, it is shown that Eqs. (5) and (6) lead to a gaugeinvariant glue angular momentum operator $[28,29]$ as

$$
\vec{J}^{g}=\int d^{3} x[\vec{x} \times(\vec{E} \times \vec{B})],
$$

where $\vec{E}$ and $\vec{B}$ are the electric and magnetic fields for gluons, respectively. Hence, the angular momentum operator in QCD can be expressed as the following gauge-invariant sum of operators [29]:

$$
\vec{J}_{\mathrm{QCD}}=\vec{J}^{q}+\vec{J}^{g}=\frac{1}{2} \vec{\Sigma}^{q}+\vec{L}^{q}+\vec{J}^{g}
$$

They represent the quark spin, the quark orbital angular momentum, and glue angular momentum, respectively. There are discussions in the literature as to whether the glue operator can be further decomposed into the spin and orbital angular momentum as in the case of the quarks [28,30-34]. We shall not address this issue in the present work.

To identify the missing dark spin from first principles, we need to measure all the three quantities in Eq. (12) using lattice QCD.

From the first term of the Eq. (9), we see that the spin contribution from quarks can be computed using the flavor singlet axial-vector operator, $\bar{\psi} \gamma^{\mu} \gamma^{5} \psi$, and it has a well-defined matrix element. There have already been a few studies on the lattice [2-5] in this regard. However, both the second term of the Eq. (9) and the term of the Eq. (11) involve the spatial coordinate $\vec{x}$. While they are natural operators for hadronic models in which the origin of the proton is prescribed, it is shown that a straightforward application of the lattice calculation of the moments of the spatial coordinate is complicated by the periodic condition of the lattice and will lead to wrong results [35]. Hence, instead of calculating the orbital angular momentum $L^{q}$ directly using lattice, we will calculate the total angular momentum $J^{q}$ for quarks and then subtract the quark spin contributions to determine $L^{q}$.
The matrix element of $\mathcal{T}^{(0 i) q, g}$ between two nucleon states can be written in terms of three form factors $\left(T_{1}, T_{2}\right.$, and $\left.T_{3}\right)$ as [29]

$$
\begin{aligned}
\left(p^{\prime}, s^{\prime}\left|\mathcal{T}^{\{0 i\} q, g}\right| p, s\right)= & \left(\frac{1}{2}\right) \bar{u}\left(p^{\prime}, s^{\prime}\right)\left[T_{1}\left(q^{2}\right)\left(\gamma^{0} \bar{p}^{i}+\gamma^{i} \bar{p}^{0}\right)\right. \\
& +\frac{1}{2 m} T_{2}\left(q^{2}\right)\left(\bar{p}^{0}\left(i \sigma^{i \alpha}\right)+\bar{p}^{i}\left(i \sigma^{0 \alpha}\right)\right) q_{\alpha} \\
& \left.+\frac{1}{m} T_{3}\left(q^{2}\right) q^{0} q^{i}\right]^{q, g} u(p, s)
\end{aligned}
$$

where $p$ and $p^{\prime}$ are the initial and final momenta of the nucleon, respectively, and $\bar{p}=\frac{1}{2}\left(p^{\prime}+p\right) . q_{\mu}=p_{\mu}^{\prime}-p_{\mu}$ is the momentum transfer to the nucleon, $m$ is the mass of the nucleon, and $u(p, s)$ is the nucleon spinor. $s$ and $s^{\prime}$ are the initial and final spins, respectively. The spinor, $u(p, s)$, satisfies the following normalization conditions:

$$
\bar{u}(p, s) u(p, s)=2 m, \quad \sum_{s} u(p, s) \bar{u}(p, s)=p+m .
$$

By substituting Eq. (13) into Eqs. (6) and (8), and then taking $q^{2} \rightarrow 0$ limit, one obtains

$$
\begin{aligned}
J^{q, g} & =\frac{1}{2}\left[T_{1}(0)+T_{2}(0)\right]^{q, g}, \\
\langle x\rangle^{q, g} & =T_{1}(0)^{q, g},
\end{aligned}
$$

where $\langle x\rangle^{q, g}=T_{1}(0)^{q, g}$ is the first moment of the momentum fraction carried by the quarks or gluons inside a nucleon. The other form factor, $T_{2}(0)^{q, g}$, can be interpreted as the anomalous gravitomagnetic moment for quarks and gluons in an analogy to the anomalous magnetic moment, $F_{2}(0)$ [36].

Since momentum is always conserved and the nucleon has a total spin of $\frac{1}{2}$, we write the momentum and angular momentum sum rules using Eqs. (12), (15), and (16) as

$$
\begin{gathered}
\langle x\rangle^{q}+\langle x\rangle^{g}=T_{1}(0)^{q}+T_{1}(0)^{g}=1, \\
J^{q}+J^{g}=\frac{1}{2}\left\{\left[T_{1}(0)+T_{2}(0)\right]^{q}+\left[T_{1}(0)+T_{2}(0)\right]^{g}\right\}=\frac{1}{2} .
\end{gathered}
$$

It is interesting to note that from Eqs. (17) and (18) one obtains that the sum of the $T_{2}(0)$ 's for the quarks and glue is zero, i.e.,

$$
T_{2}(0)^{q}+T_{2}(0)^{g}=0 .
$$

The vanishing of $T_{2}(0)$ in the context of a spin- $1 / 2$ particle was first derived classically from the post-Newtonian manifestation of the equivalence principle [37]. More recently, this has been proven by Brodsky et al. [38] for 
composite systems from the light-cone Fock space representation.

Since we are going to evaluate $J^{q, g}$ (or $L^{q, g}$ ) and $\langle x\rangle^{q, g}$ in this work, it is clear from Eqs. (15) and (16) that we need to compute both $T_{1}(0)$ and $T_{2}(0)$. However, $T_{2}(0)$ cannot be computed directly at $q=0$ because the $T_{2}$ form factor in Eq. (13) is proportional to $q$. Instead, we shall compute $T_{1}\left(q^{2}\right)$ and $T_{2}\left(q^{2}\right)$ separately at some $q^{2} \neq 0$ values [11] and then separately extrapolate them to $q^{2} \rightarrow 0$ for both the quark and glue contributions.

\section{LATTICE FORMALISM}

\section{A. Operators and matrix elements in Euclidean space-time}

To carry out lattice calculations, we use the PauliSakurai convention [39-41] for the $\gamma$ matrices in
Euclidean space-time. We can then write the energymomentum tensor for quarks and gluons as

$$
\mathcal{T}_{\{4 i\}}^{q(E)}=(-1) \frac{i}{4} \sum_{f} \bar{\psi}_{f}\left[\gamma_{4} \vec{D}_{i}+\gamma_{i} \vec{D}_{4}-\gamma_{4} \stackrel{\leftarrow}{D}_{i}-\gamma_{i} \stackrel{\leftarrow}{D}_{4}\right] \psi_{f},
$$

$$
\mathcal{T}_{\{4 i\}}^{g(E)}=(+i)\left[-\frac{1}{2} \sum_{k=1}^{3} 2 \operatorname{Tr}^{\text {color }}\left[G_{4 k} G_{k i}+G_{i k} G_{k 4}\right]\right] .
$$

The matrix elements for both quarks and gluons transform in a similar manner as

$$
\begin{aligned}
\left\langle p^{\prime}, s^{\prime}\left|\mathcal{T}_{\{4 i\}}^{q, g(E)}\right| p, s\right\rangle= & \left(\frac{1}{2}\right) \bar{u}^{(E)}\left(p^{\prime}, s^{\prime}\right)\left[T_{1}\left(-q^{2}\right)\left(\gamma_{4} \bar{p}_{i}+\gamma_{i} \bar{p}_{4}\right)-\frac{1}{2 m} T_{2}\left(-q^{2}\right)\left(\bar{p}_{4} \sigma_{i \alpha} q_{\alpha}+\bar{p}_{i} \sigma_{4 \alpha} q_{\alpha}\right)\right. \\
& \left.-\frac{i}{m} T_{3}\left(-q^{2}\right) q_{4} q_{i}\right]^{q, g} u^{(E)}(p, s),
\end{aligned}
$$

where the normalization conditions are

$$
\begin{aligned}
\bar{u}^{(E)}(p, s) u^{(E)}(p, s) & =1, \\
\sum_{s} u^{(E)}(p, s) \bar{u}^{(E)}(p, s) & =\frac{p+m}{2 m},
\end{aligned}
$$

and the lhs of Eqs. (13) and (22) are related by

$$
\frac{\left(p^{\prime}, s^{\prime}\right.}{\sqrt{2 m}}\left|\mathcal{T}^{\{0 i\} q, g \mid}\right| \frac{p, s)}{\sqrt{2 m}} \longleftrightarrow\left\langle p^{\prime}, s^{\prime}\left|\mathcal{T}_{\{4 i\}}^{q, g(E)}\right| p, s\right\rangle .
$$

From now on, we will consider the Euclidean operators only and drop the superscript, $E$.

\section{B. Quark energy-momentum tensor operator}

We discretize $\mathcal{T}_{\{4 i\}}^{q}$ by using the relations for right and left derivatives in the lattice [42],

$$
\begin{aligned}
\vec{D}_{\mu} \psi^{L}(x)= & \frac{1}{2 a}\left[U_{\mu}(x) \psi^{L}\left(x+a_{\mu}\right)\right. \\
& \left.-U_{\mu}^{\dagger}\left(x-a_{\mu}\right) \psi^{L}\left(x-a_{\mu}\right)\right], \\
\bar{\psi}^{L}(x) \overleftarrow{D}_{\mu}= & \frac{1}{2 a}\left[\bar{\psi}^{L}\left(x+a_{\mu}\right) U_{\mu}^{\dagger}(x)\right. \\
& \left.-\bar{\psi}^{L}\left(x-a_{\mu}\right) U_{\mu}\left(x-a_{\mu}\right)\right],
\end{aligned}
$$

where $a$ is the lattice spacing, $\psi^{L}$ 's are the Lattice quark field operators, and $U$ 's are the gauge links. Using the relations in Eqs. (25) and (26), we get

$$
\begin{aligned}
\mathcal{T}_{\{4 i\}}^{q}(x)= & \frac{-i}{8 a}\left[\bar{\psi}_{f}(x) \gamma_{4} U_{i}(x) \psi_{f}\left(x+a_{i}\right)-\bar{\psi}_{f}(x) \gamma_{4} U_{i}^{\dagger}\left(x-a_{i}\right) \psi_{f}\left(x-a_{i}\right)\right. \\
& +\bar{\psi}_{f}\left(x-a_{i}\right) \gamma_{4} U_{i}\left(x-a_{i}\right) \psi_{f}(x)-\bar{\psi}_{f}\left(x+a_{i}\right) \gamma_{4} U_{i}^{\dagger}(x) \psi_{f}(x) \\
& +\bar{\psi}_{f}(x) \gamma_{i} U_{4}(x) \psi_{f}\left(x+a_{4}\right)-\bar{\psi}_{f}(x) \gamma_{i} U_{4}^{\dagger}\left(x-a_{4}\right) \psi_{f}\left(x-a_{4}\right) \\
& \left.+\bar{\psi}_{f}\left(x-a_{4}\right) \gamma_{i} U_{4}\left(x-a_{4}\right) \psi_{f}(x)-\bar{\psi}_{f}\left(x+a_{4}\right) \gamma_{i} U_{4}^{\dagger}(x) \psi_{f}(x)\right] .
\end{aligned}
$$

\section{Glue energy-momentum tensor operator}

It is well known that gauge operators obtained from the link variables are very noisy due to the large fluctuations in high-frequency modes. A preliminary study of the glue momentum fraction in the nucleon on a quenched lattice concluded that configurations in the order of several hundred thousands might be needed for a precise signal 
[43]. This is a tall order for dynamical fermion calculations. On the other hand, a smeared operator will improve the signal, and hypercubic (HYP) smearing has been applied to calculate the glue momentum fraction in the pion with reasonable precision [44].

Because of the exponentially local nature of the overlap Dirac operator through chiral smearing [45-47], the subdimensional long-range order of the topological structure has been discovered [48,49] with the help of the local topological charge operator derived from the massless overlap Dirac operator, i.e., $q(x)=\operatorname{Tr} \gamma_{5}\left(1-\frac{1}{2} D_{\text {ov }}(x, x)\right)$ [50-53]. Prompted by this success, it is shown that the gauge-field tensor can be similarly derived from the massless overlap operator $D_{\text {ov }}[24,25]$,

$$
\operatorname{Tr}_{s}\left[\sigma_{\mu \nu} D_{\mathrm{ov}}(x, x)\right]=c_{T} a^{2} G_{\mu \nu}(x)+\mathcal{O}\left(a^{3}\right),
$$

where $\operatorname{Tr}_{s}$ is the trace over the spin. $c_{T}=0.11157$ is the proportional constant at the continuum limit for the parameter $\kappa=0.19$ in the Wilson kernel of the overlap operator that is used in this work. The glue energymomentum tensor in Eq. (21) constructed with this gauge-field tensor was used in calculating the glue momentum fraction, $\langle x\rangle_{g}$, which resulted in a first observation with a much better signal [54].

We shall use the energy-momentum tensor with the noise-estimated gauge-field tensor as defined in Eq. (21) from the overlap Dirac operator to calculate the glue momentum and angular momentum in the nucleon.

\section{Two-point correlation functions}

To obtain $T_{1}(0)^{q, g}$ and $T_{2}(0)^{q, g}$, we first need to calculate the two-point and three-point functions (both polarized and unpolarized) for protons (neutrons). The two-point function is defined (with the color indices suppressed) as

$G_{\alpha \beta}^{N N}\left(\vec{p}, t ; t_{0}\right)=\sum_{\vec{x}} e^{-i \vec{p} \cdot\left(\vec{x}-\vec{x}_{0}\right)}\left\langle 0\left|\mathrm{~T}\left[\chi_{\alpha}(\vec{x}, t) \bar{\chi}_{\beta}\left(\vec{x}_{0}, t_{0}\right)\right]\right| 0\right\rangle$,

where $t$ is the nucleon sink time, $\vec{p}$ is the momentum of the nucleon, and $x_{0}$ is the source position. The interpolating fields, $\chi$ 's, for nucleons that we use are given by $[55,56]$

$$
\begin{gathered}
\chi_{\gamma}(x)=\epsilon_{a b c} \psi_{\alpha}^{T(u) a}(x)\left(C \gamma_{5}\right)_{\alpha \beta} \psi_{\beta}^{(d) b}(x) \psi_{\gamma}^{(u) c}(x), \\
\bar{\chi}_{\gamma^{\prime}}(x)=-\epsilon_{d e f} \bar{\psi}_{\gamma^{\prime}}^{(u) f}(x) \bar{\psi}_{\rho}^{(d) e}(x)\left(\gamma_{5} C\right)_{\rho \sigma} \bar{\psi}_{\sigma}^{T(u) d}(x),
\end{gathered}
$$

where $u$ and $d$ stand for up and down quarks, respectively. $C=\gamma_{2} \gamma_{4}$ is the charge conjugation operator with the Pauli-Sakurai $\gamma$ matrices. The letters, $a, b, \ldots$, stand for the color indices. The Greek letters, $\alpha, \beta, \ldots$, are the spin indices.

Upon Grassmann integration for Eq. (29), we obtain the unpolarized/polarized proton two-point function on a gauge configuration $U$ as

$$
\begin{aligned}
\operatorname{Tr}\left[\Gamma^{\mathrm{unpol}, \mathrm{pol}} G^{N N}\left(\vec{p}, t ; t_{0} ; U\right)\right]= & \sum_{\vec{x}} e^{-i \vec{p} \cdot\left(\vec{x}-\vec{x}_{0}\right)} \epsilon_{a b c} \epsilon_{d e f}\left\{\operatorname{Tr}\left[\Gamma^{\mathrm{unpol}, \mathrm{pol}} S^{(u) a d}\left(x, x_{0} ; U\right)\right] \operatorname{Tr}\left[\tilde{S}^{(u) b e}\left(x, x_{0} ; U\right) S^{(d) c f}\left(x, x_{0} ; U\right)\right]\right. \\
& \left.+\operatorname{Tr}\left[\Gamma^{\mathrm{unpol}, \mathrm{pol}} S^{(u) a d}\left(x, x_{0} ; U\right) \tilde{S}^{(d) b e}\left(x, x_{0} ; U\right) S^{(u) c f}\left(x, x_{0} ; U\right)\right]\right\} \\
= & \sum_{\vec{x}} e^{-i \vec{p} \cdot\left(\vec{x}-\vec{x}_{0}\right)} N^{\mathrm{unpol}, \mathrm{pol}}[x ; U],
\end{aligned}
$$

where $\Gamma^{\text {unpol,pol }}$ are the unpolarized/polarized projection operators and $N^{\text {unpol,pol }}[x ; U]$ stands for the trace part of two-point functions (without the Fourier factor). $S^{(f)}(x, y ; U)$ is the quark propagator with flavor $f$ from the point $y$ to $x$ on the gauge configuration $U$, and

$$
\tilde{S}=\left(C \gamma_{5}\right)^{-1} S^{T}\left(C \gamma_{5}\right),
$$

On the other hand, if we insert a complete set of energy eigenstates in Eq. (29), and take the trace with the unpolarized projection operator, then at a large time separation, we get the two-point functions for nucleons as

$$
\begin{aligned}
& \operatorname{Tr}\left[\Gamma^{\text {unpol }} G^{N N}\left(\vec{p}, t ; t_{0}\right)\right] \stackrel{\left(t-t_{0} \gg 1 / \Delta E\right)}{\longrightarrow} \frac{a^{6}}{(2 k)^{3}}|\phi(p)|^{2} \\
& \times e^{-i \vec{p} \cdot \vec{x}_{0}} \frac{E_{p}+m}{E_{p}} e^{-E_{p}\left(t-t_{0}\right)},
\end{aligned}
$$

where $\kappa$ is the hopping parameter, $m$ is the mass of the nucleon, and $E_{p}$ is its ground-state energy. $\Delta E$ is the energy gap between the ground state and first excited state. $\phi(p)$ is the vacuum-to-nucleon transition matrix element due to the interpolation field $\chi$, and we treat it as a function of $p$ to account for the possible $p$-dependent lattice systematics.

\section{E. Three-point correlation functions}

The three-point functions for $\mathcal{T}_{\{4 i\}}^{q, g}$ (or any generic operator) is defined as

$$
\begin{aligned}
& G_{\alpha \beta}^{N \mathcal{T}_{4 i} N}\left(\vec{p}^{\prime}, t_{2} ; \vec{q}, t_{1} ; \vec{p}, t_{0}\right) \\
& =\sum_{\vec{x}_{1}, \vec{x}_{2}} e^{-i \vec{p}^{\prime} \cdot\left(\vec{x}_{2}-\vec{x}_{1}\right)} e^{-i \vec{p} \cdot\left(\vec{x}_{1}-\vec{x}_{0}\right)} \\
& \quad \times\left\langle 0\left|\mathrm{~T}\left[\chi_{\alpha}\left(\vec{x}_{2}, t_{2}\right) \mathcal{T}_{\{4 i\}}\left(\vec{x}_{1}, t_{1}\right) \bar{\chi}_{\beta}\left(\vec{x}_{0}, t_{0}\right)\right]\right| 0\right\rangle,
\end{aligned}
$$


where $t_{2}$ is the nucleon sink time, $t_{1}$ is the current insertion time, and $t_{0}$ is the nucleon source time. $\vec{p}$ and $\vec{p}^{\prime}$ are the initial and final momenta of the nucleon, respectively, and $\vec{q}=\vec{p}^{\prime}-\vec{p}$ is the momentum transfer.

By inserting a complete set of energy eigenstates in Eq. (35) and then taking the trace with $\Gamma^{\text {unpol,pol }}$, we get the threepoint functions as

$$
\begin{aligned}
& \operatorname{Tr}\left[\Gamma^{\text {unpol,pol }} G^{(f) N \mathcal{T}_{4 i} N}\left(\vec{p}^{\prime}, t_{2} ; \vec{q}, t_{1} ; \vec{p}, t_{0}\right)\right] \stackrel{\left(t_{1}-t_{0}\right),\left(t_{2}-t_{1}\right) \gg 1 / \Delta E}{\longrightarrow} \\
& \frac{a^{6}}{(2 \kappa)^{3}} \phi(p) \phi\left(p^{\prime}\right) e^{-i \vec{p} \cdot \vec{x}_{0}} \frac{1}{4} \frac{1}{2 \kappa} \frac{1}{E_{p} E_{p^{\prime}}} e^{-E_{p}\left(t_{2}-t_{1}\right)} e^{-E_{p^{\prime}}\left(t_{1}-t_{0}\right)}\left[a_{1} T_{1}\left(q^{2}\right)+a_{2} T_{2}\left(q^{2}\right)+a_{3} T_{3}\left(q^{2}\right)\right],
\end{aligned}
$$

where $a_{i}$ 's are constant coefficients that depend upon the momentum and energy of the proton and, therefore, are known a priori. For the unpolarized case, they can be written as

$$
\begin{aligned}
a_{1}= & \left(p_{i}^{\prime}+p_{i}\right)\left(\left(E_{p}+m\right)\left(E_{p^{\prime}}+m\right)+p_{j}^{\prime} p_{j}\right)+\left(E_{p^{\prime}}+E_{p}\right)\left(p_{i}^{\prime}\left(E_{p}+m\right)+p_{i}\left(E_{p^{\prime}}+m\right)\right), \\
a_{2}= & -\frac{1}{2 m}\left\{\left(E_{p}+m\right)\left(\left(E_{p^{\prime}}^{2}-E_{p}^{2}\right) p_{i}^{\prime}+\left(p_{i}^{\prime}+p_{i}\right) q_{j} p_{j}^{\prime}\right)\right. \\
& \left.-\left(E_{p^{\prime}}+m\right)\left(\left(E_{p^{\prime}}^{2}-E_{p}^{2}\right) p_{i}-\left(p_{i}^{\prime}+p_{i}\right) q_{j} p_{j}\right)+\left(E_{p^{\prime}}+E_{p}\right)\left(p_{j}^{\prime} p_{i} q_{j}-p_{i}^{\prime} p_{j} q_{j}\right)\right\}, \\
a_{3}= & \frac{2}{m}\left(E_{p^{\prime}}-E_{p}\right) q_{i}\left(\left(E_{p}+m\right)\left(E_{p^{\prime}}+m\right)-p_{j}^{\prime} p_{j}\right),
\end{aligned}
$$

and for the polarized case, they are

$$
\begin{aligned}
a_{1}= & (-i) \epsilon_{i j l}\left(E_{p^{\prime}}+E_{p}\right)\left(p_{j}^{\prime}\left(E_{p}+m\right)-p_{j}\left(E_{p^{\prime}}+m\right)\right)+\epsilon_{k j l}\left(p_{i}^{\prime}+p_{i}\right) p_{j}^{\prime} p_{k}, \\
a_{2}= & \frac{-i}{2 m}\left\{\left(E_{p}+m\right)\left(E_{p^{\prime}}+m\right) \epsilon_{i j l}\left(E_{p^{\prime}}+E_{p}\right) q_{j}\right. \\
& -\left(\left(E_{p}+m\right) p_{j}^{\prime}+\left(E_{p^{\prime}}+m\right) p_{j}\right)\left(\epsilon_{i j l}\left(E_{p^{\prime}}^{2}-E_{p}^{2}\right)+\epsilon_{k j l}\left(p_{i}^{\prime}+p_{i}\right) q_{k}\right) \\
& \left.-\left(\epsilon_{i j k}\left(E_{p^{\prime}}+E_{p}\right)\left(p_{l}^{\prime} p_{k} q_{j}+p_{j}^{\prime} p_{l} q_{k}\right)+\epsilon_{i j l}\left(E_{p^{\prime}}+E_{p}\right) p_{k}^{\prime} p_{k} q_{j}\right)\right\}, \\
a_{3}= & \frac{-2 i}{m} \epsilon_{k j l}\left(E_{p^{\prime}}-E_{p}\right) q_{i} p_{j}^{\prime} p_{k} .
\end{aligned}
$$

Here, the subscript $i$ stands for the spatial direction of the energy-momentum operator, and $l$ is the direction of polarization of the nucleon.

The three-point functions for quarks have two topologically distinct contributions in the path-integral diagrams: one from connected insertions (CI) and the other from disconnected insertions (DI) [57-59] (see Fig. 1). They arise purely out of Wick contractions, and it needs to be stressed that they are not Feynman diagrams in perturbation theory. In the case of CI, quark/antiquark fields from the operator are contracted with the quark/antiquark fields of the proton interpolating fields. In the case of DI, the quark/antiquark fields from the operator contract themselves to form a current loop, as in the case of vacuum polarization.

Though not shown in the figure, the loop is in fact connected with the proton propagator through the gauge background fluctuations. In practice, the uncorrelated part of the loop and the proton propagator is subtracted. The disconnected insertion refers to the fact that the quark lines are disconnected.

For currents with up and down quarks, we have contributions from both CI and DI, and with strange quarks, we have DI only.

The current loop for our operator $\mathcal{T}_{\{4 i\}}$ with a quark flavor $f$ is given by

$$
\begin{aligned}
L\left[t_{1}, \vec{q} ; U\right]= & (-1) \frac{-i}{8 a} \sum_{\vec{x}_{1}} e^{i \vec{q} \cdot\left(\vec{x}_{1}-\vec{x}_{0}\right)}\left\{\operatorname{Tr}\left[S^{(f) m n}\left(x_{1}+a_{i}, x_{1} ; U\right) \gamma_{4} U_{i}^{n m}\left(x_{1}\right)\right]-\operatorname{Tr}\left[S^{(f) m n}\left(x_{1}-a_{i}, x_{1} ; U\right) \gamma_{4} U_{i}^{\dagger n m}\left(x_{1}-a_{i}\right)\right]\right. \\
& +\operatorname{Tr}\left[S^{(f) m n}\left(x_{1}, x_{1}-a_{i}\right) \gamma_{4} U_{i}^{n m}\left(x_{1}-a_{i}\right)\right]-\operatorname{Tr}\left[S^{(f) m n}\left(x_{1}, x_{1}+a_{i} ; U\right) \gamma_{4} U_{i}^{\dagger n m}\left(x_{1}\right)\right] \\
& +\operatorname{Tr}\left[S^{(f) m n}\left(x_{1}+a_{4}, x_{1} ; U\right) \gamma_{i} U_{4}^{n m}\left(x_{1}\right)\right]-\operatorname{Tr}\left[S^{(f) m n}\left(x_{1}-a_{4}, x_{1} ; U\right) \gamma_{i} U_{4}^{\dagger n m}\left(x_{1}-a_{4}\right)\right] \\
& +\operatorname{Tr}\left[S^{(f) m n}\left(x_{1}, x_{1}-a_{4} ; U\right) \gamma_{i} U_{4}^{n m}\left(x_{1}-a_{4}\right)\right]-\operatorname{Tr}\left[S^{(f) m n}\left(x_{1}, x_{1}+a_{4} ; U\right) \gamma_{i} U_{4}^{\dagger n m}\left(x_{1}\right)\right\} \\
= & +i \\
\hline a & \sum_{\vec{x}_{1}} e^{i \vec{q} \cdot\left(\vec{x}_{1}-\vec{x}_{0}\right)} L\left[x_{1} ; U\right]
\end{aligned}
$$




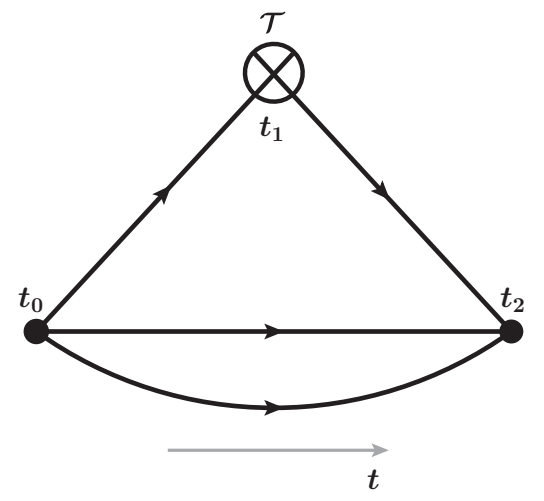

(a)

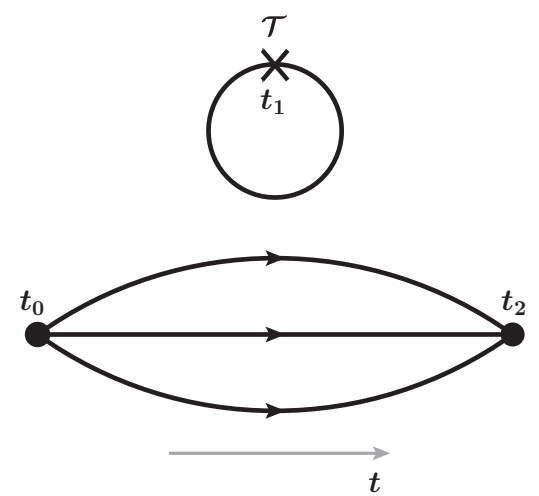

(b)

FIG. 1. Quark line diagrams of the three-point function with current insertion in the Euclidean path integral formalism. (a) Connected insertions (CI) and (b) disconnected insertions (DI).

where $L\left[x_{1} ; U\right]$ is the trace part of the current loop. The gauge-averaged DI three-point functions can then be written as

$$
\begin{aligned}
\operatorname{Tr}\left[\Gamma^{\text {unpol,pol }} G^{(f) N \mathcal{T}_{4 i} N}\left(\vec{p}^{\prime}, t_{2} ; \vec{q}, t_{1} ; \vec{p}, t_{0}\right)\right]_{\mathrm{DI}} \\
=\left\langle\operatorname{Tr}\left[\Gamma^{\mathrm{unpol}, \mathrm{pol}} G^{N N}\left(\vec{p}^{\prime}, t_{2} ; t_{0} ; U\right)\right] \times L\left[t_{1}, \vec{q} ; U\right]\right\rangle \\
\quad-\left\langle\operatorname{Tr}\left[\Gamma^{\text {unpol,pol }} G^{N N}\left(\vec{p}^{\prime}, t_{2} ; t_{0} ; U\right)\right]\right\rangle \times\left\langle L\left[t_{1}, \vec{q} ; U\right]\right\rangle,
\end{aligned}
$$

where $\langle\cdots\rangle$ denotes the average over the gauge ensemble. It is important to note that the three-point functions for gluons have the similar form as DI.

The computation of the CI is relatively straightforward. We shall use the sequential source technique [60-62] for CI. This fixes the source point $t_{0}$ and the sink time slice $t_{2}$. However, the computation of the DI is numerically challenging as it contains not only the usual propagators from the source, $x_{0}$, to any point, $x$, but also the propagators from any insertion position, $x_{1}$, to any other lattice points.
This would require inverting the fermion matrix at each point of the lattice to construct the all-to-all propagators. Naively, this entails inversion of a million by million $\left(\sim 16^{3} \times 24 \times 3 \times 4\right)$ sparse matrix for our $16^{3} \times 24$ lattice ( 3 and 4 being the number of color and spin indices, respectively) on each gauge configuration. This is unattainable by using the computing powers of today's supercomputers. Instead, we shall compute it with the stochastic method. Specifically, we adopt the complex $Z_{2}$ noise [63] for the estimation together with unbiased subtraction [64] to reduce variance. The detailed description of the method and the usefulness of discrete symmetries that are applicable to both DI and glue will be presented in Sec. IV B.

\section{F. Ratios of correlation functions}

After evaluating two-point and three-point correlation functions, we take the following ratios between three-point to two-point functions, which at a large time separation involve the combinations of $T_{1}\left(q^{2}\right), T_{2}\left(q^{2}\right)$, and $T_{3}\left(q^{2}\right)$ :

$$
\begin{aligned}
& \frac{\operatorname{Tr}\left[\Gamma^{\text {unpol, pol }} G^{N \mathcal{T}_{4 i} N}\left(\vec{p}^{\prime}, t_{2} ; \vec{q}, t_{1} ; \vec{p}, t_{0}\right)\right]}{\operatorname{Tr}\left[\Gamma^{\mathrm{unpol}} G^{N N}\left(\vec{p}^{\prime}, t_{2} ; t_{0}\right)\right]} \times \sqrt{\frac{\operatorname{Tr}\left[\Gamma^{\mathrm{unpol}} G^{N N}\left(\vec{p}, t_{2}-t_{1}+t_{0} ; t_{0}\right)\right]}{\operatorname{Tr}\left[\Gamma^{\text {unpol }} G^{N N}\left(\vec{p}^{\prime}, t_{2}-t_{1}+t_{0} ; t_{0}\right)\right]}} \\
& \quad \times \sqrt{\frac{\operatorname{Tr}\left[\Gamma^{\text {unpol }} G^{N N}\left(\vec{p}^{\prime}, t_{1} ; t_{0}\right)\right]}{\operatorname{Tr}\left[\Gamma^{\text {unpol }} G^{N N}\left(\vec{p}, t_{1} ; t_{0}\right)\right]} \times \frac{\operatorname{Tr}\left[\Gamma^{\text {unpol }} G^{N N}\left(\vec{p}^{\prime}, t_{2} ; t_{0}\right)\right]}{\operatorname{Tr}\left[\Gamma^{\text {unpol }} G^{N N}\left(\vec{p}, t_{2} ; t_{0}\right)\right]}} \stackrel{\left(t_{1}-t_{0}\right),\left(t_{2}-t_{1}\right) \gg 1 / \Delta E}{\longrightarrow} \frac{\left[a_{1} T_{1}\left(q^{2}\right)+a_{2} T_{2}\left(q^{2}\right)+a_{3} T_{3}\left(q^{2}\right)\right]}{4 \sqrt{E_{p^{\prime}}\left(E_{p^{\prime}}+m\right) E_{p}\left(E_{p}+m\right)}} .
\end{aligned}
$$

Since $T_{1}^{q}\left(q^{2}\right)$ and $T_{2}^{q}\left(q^{2}\right)$ for the CI are shown to have quite different $q^{2}$ behavior [10-12], we need to separately extrapolate $T_{1}\left(q^{2}\right)$ and $T_{2}\left(q^{2}\right)$ to $q^{2} \longrightarrow 0$ (we shall do this both for CI and DI as well as for the glue contribution). To achieve this, we shall combine results from different kinematics for both the polarized and unpolarized three-point functions into the ratios in Eq. (41) at a particular $q^{2}$. The ratios then appear as different combinations in different $a_{i}$ 's from which one can separate $T_{1}\left(q^{2}\right), T_{2}\left(q^{2}\right)$, and $T_{3}\left(q^{2}\right)$ and then extrapolate $T_{1}\left(q^{2}\right)$ and $T_{2}\left(q^{2}\right)$ in $q^{2}$ to obtain $T_{1}(0)$ and $T_{2}(0)$. The procedure to extract $T_{1}\left(q^{2}\right), T_{2}\left(q^{2}\right)$, and $T_{3}\left(q^{2}\right)$ is discussed in detail in Sec. VI. 


\section{Special case: $\vec{p}^{\prime}=0$ or $\vec{p}=0$}

If we consider the special case with $\vec{p}^{\prime}=0$ for the polarized three-point functions, we obtain

$$
\frac{\operatorname{Tr}\left[\Gamma_{l}^{\mathrm{pol}} G^{N \mathcal{T}_{4 i} N}\left(\overrightarrow{0}, t_{2} ; \vec{q}, t_{1} ;-\vec{q}, t_{0}\right)\right]}{\operatorname{Tr}\left[\Gamma^{\mathrm{unpol}} G^{N N}\left(\overrightarrow{0}, t_{2} ; t_{0}\right)\right]} \cdot \frac{\operatorname{Tr}\left[\Gamma^{\mathrm{unpol}} G^{N N}\left(\overrightarrow{0}, t_{1} ; t_{0}\right)\right]}{\operatorname{Tr}\left[\Gamma^{\mathrm{unpol}} G^{N N}\left(\vec{q}, t_{1} ; t_{0}\right)\right]} \stackrel{\left(t_{1}-t_{0}\right),\left(t_{2}-t_{1}\right) \gg 1 / \Delta E}{\longrightarrow} \frac{-i}{4} \epsilon_{i j l} q_{j}\left[T_{1}+T_{2}\right]\left(q^{2}\right),
$$

where $\left[T_{1}+T_{2}\right]\left(q^{2}\right)=T_{1}\left(q^{2}\right)+T_{2}\left(q^{2}\right)$. Similarly, if we consider $\vec{p}=0$, we get

$$
\frac{\operatorname{Tr}\left[\Gamma_{l}^{\mathrm{pol}} G^{N \mathcal{T}_{i i} N}\left(\vec{q}, t_{2} ; \vec{q}, t_{1} ; \overrightarrow{0}, t_{0}\right)\right]}{\operatorname{Tr}\left[\Gamma^{\mathrm{unpol}} G^{N N}\left(\vec{q}, t_{2} ; t_{0}\right)\right]} \cdot \frac{\operatorname{Tr}\left[\Gamma^{\mathrm{unpol}} G^{N N}\left(\vec{q}, t_{1} ; t_{0}\right)\right]}{\operatorname{Tr}\left[\Gamma^{\mathrm{unpol}} G^{N N}\left(\overrightarrow{0}, t_{1} ; t_{0}\right)\right]} \stackrel{\left(t_{1}-t_{0}\right),\left(t_{2}-t_{1}\right) \gg 1 / \Delta E}{\longrightarrow} \frac{-i}{4} \frac{E_{p^{\prime}}+m}{m} \epsilon_{i j l} q_{j}\left[T_{1}+T_{2}\right]\left(q^{2}\right) .
$$

In the unpolarized case, the three-point functions vanish when either $\vec{p}^{\prime}=0$ or $\vec{p}=0$.

We shall use the Eqs. (42) and (43) to check the extracted values of $T_{1}\left(q^{2}\right)$ and $T_{2}\left(q^{2}\right)$ by comparing $\left[T_{1}\left(q^{2}\right)+T_{2}\left(q^{2}\right)\right]$ against $\left[T_{1}+T_{2}\right]\left(q^{2}\right)$ obtained directly at comparable $q^{2}$.

\section{Special case: $\vec{p}^{\prime}=\vec{p}$}

If we consider the forward matrix element in which $\vec{p}^{\prime}=\vec{p}$ and take the following ratio with unpolarized three-point functions, we obtain

$$
\frac{\operatorname{Tr}\left[\Gamma^{\text {unpol }} G^{N \mathcal{T}_{4 i} N}\left(\vec{p}^{\prime}, t_{2} ; \overrightarrow{0}, t_{1} ; \vec{p}^{\prime}, t_{0}\right)\right]}{\operatorname{Tr}\left[\Gamma^{\text {unpol }} G^{N N}\left(\vec{p}^{\prime}, t_{2}\right)\right]} \stackrel{\left(t_{1}-t_{0}\right),\left(t_{2}-t_{1}\right) \gg 1 / \Delta E}{\longrightarrow} T_{1}(0)=\langle x\rangle .
$$

For the polarized case, the above ratio vanishes. We see that Eq. (44) gives us the first moment of the parton distribution, or $T_{1}(0)$, directly, which has been calculated on the same lattice in Ref. [41]. The value of $T_{1}(0)$ obtained from Eq. (44) can be checked against the $q^{2}$ extrapolated value of $T_{1}(0)$ obtained from Eq. (41) or vice versa.

Since Eq. (44) allows a direct determination of $T_{1}(0)$ without requiring one to perform $q^{2} \rightarrow 0$ extrapolation, this results in a much clearer signal for $T_{1}(0)$ as compared to that obtained from Eq. (41). The results from both the methods are presented in Sec. VIII, and, in fact, we shall combine the former one with the extrapolated value of $T_{2}(0)$ from Eq. (41) in order to construct $J^{q, g}$. Please note that these two methods for determining $T_{1}(0)$ are independent of each other since, in the $q^{2} \rightarrow 0$ extrapolation method, we do not take into account the $q^{2}=0$ data point that comes from Eq. (44). As mentioned earlier, this provides us a check for the value of $T_{1}(0)$.

\section{Ratios for disconnected insertions}

As mentioned in Sec. III E, the sink time is fixed for CI. But in DI, the sink time need not be fixed, and we can sum over the insertion time, $t_{1}$, between the source and the sink time, i.e., from $t_{1}=t_{0}+1$ to $t_{2}-1$ to gain more statistics $[2,41,65,66]$. Moreover, such summation helps in suppressing the excited state contamination $[41,65]$. This is similarly true for gluons. Then, the corresponding ratios at large time separation for Eqs. (41), (42), (43), and (44) become

$$
\begin{aligned}
& \sum_{t_{1}=t_{0}+1}^{t_{2}-1} \frac{\operatorname{Tr}\left[\Gamma^{\text {unpol,pol }} G^{N \mathcal{T}_{4 i} N}\left(\vec{p}^{\prime}, t_{2} ; \vec{q}, t_{1} ; \vec{p}, t_{0}\right)\right]}{\operatorname{Tr}\left[\Gamma^{\text {unpol }} G^{N N}\left(\vec{p}^{\prime}, t_{2} ; t_{0}\right)\right]} \times \sqrt{\frac{\operatorname{Tr}\left[\Gamma^{\text {unpol }} G^{N N}\left(\vec{p}, t_{2}-t_{1}+t_{0} ; t_{0}\right)\right]}{\operatorname{Tr}\left[\Gamma^{\text {unpol }} G^{N N}\left(\vec{p}^{\prime}, t_{2}-t_{1}+t_{0} ; t_{0}\right)\right]}} \\
& \quad \times \sqrt{\frac{\operatorname{Tr}\left[\Gamma^{\text {unpol }} G^{N N}\left(\vec{p}^{\prime}, t_{1} ; t_{0}\right)\right]}{\operatorname{Tr}\left[\Gamma^{\text {unpol }} G^{N N}\left(\vec{p}, t_{1} ; t_{0}\right)\right]} \cdot \frac{\operatorname{Tr}\left[\Gamma^{\text {unpol }} G^{N N}\left(\vec{p}^{\prime}, t_{2} ; t_{0}\right)\right]}{\operatorname{Tr}\left[\Gamma^{\text {unpol }} G^{N N}\left(\vec{p}, t_{2} ; t_{0}\right)\right]} \stackrel{\left(t_{1}\right),\left(t_{2}-t_{1}\right) \gg 1 / \Delta E}{\longrightarrow} \frac{\left[a_{1} T_{1}\left(q^{2}\right)+a_{2} T_{2}\left(q^{2}\right)+a_{3} T_{3}\left(q^{2}\right)\right]}{4 \sqrt{E_{p^{\prime}}\left(E_{p^{\prime}}+m\right) E_{p}\left(E_{p}+m\right)}} \times t_{2}+\text { const },}
\end{aligned}
$$

$$
\sum_{t_{1}=t_{0}+1}^{t_{2}-1} \frac{\operatorname{Tr}\left[\Gamma_{l}^{\mathrm{pol}} G^{N \mathcal{T}_{4 i} N}\left(\overrightarrow{0}, t_{2} ; \vec{q}, t_{1} ;-\vec{q}, t_{0}\right)\right]}{\operatorname{Tr}\left[\Gamma^{\mathrm{unpol}} G^{N N}\left(\overrightarrow{0}, t_{2} ; t_{0}\right)\right]} \cdot \frac{\operatorname{Tr}\left[\Gamma^{\mathrm{unpol}} G^{N N}\left(\overrightarrow{0}, t_{1} ; t_{0}\right)\right]}{\operatorname{Tr}\left[\Gamma^{\mathrm{unpol}} G^{N N}\left(\vec{q}, t_{1} ; t_{0}\right)\right]} \stackrel{\left(t_{1}-t_{0}\right),\left(t_{2}-t_{1}\right) \gg 1 / \Delta E}{\longrightarrow} \frac{-i}{4} \epsilon_{i j l} q_{j}\left[T_{1}+T_{2}\right]\left(q^{2}\right) \times t_{2}+\text { const },
$$




$$
\sum_{t_{1}=t_{0}+1}^{t_{2}-1} \frac{\operatorname{Tr}\left[\Gamma_{l}^{\mathrm{pol}} G^{N \mathcal{T}_{4 i} N}\left(\vec{q}, t_{2} ; \vec{q}, t_{1} ; \overrightarrow{0}, t_{0}\right)\right]}{\operatorname{Tr}\left[\Gamma^{\mathrm{unpol}} G^{N N}\left(\vec{q}, t_{2} ; t_{0}\right)\right]} \cdot \frac{\operatorname{Tr}\left[\Gamma^{\mathrm{unpol}} G^{N N}\left(\vec{q}, t_{1} ; t_{0}\right)\right]}{\operatorname{Tr}\left[\Gamma^{\mathrm{unpol}} G^{N N}\left(\overrightarrow{0}, t_{1} ; t_{0}\right)\right]} \stackrel{\left(t_{1}-t_{0}\right),\left(t_{2}-t_{1}\right) \gg 1 / \Delta E}{\longrightarrow} \frac{-i}{4} \frac{E_{p^{\prime}}+m}{m} \epsilon_{i j l} q_{j}\left[T_{1}+T_{2}\right]\left(q^{2}\right) \times t_{2}+\text { const },
$$

$$
\sum_{t_{1}=t_{0}+1}^{t_{2}-1} \frac{\operatorname{Tr}\left[\Gamma^{\mathrm{unpol}} G^{N \mathcal{T}_{4 i} N}\left(\vec{p}^{\prime}, t_{2} ; \overrightarrow{0}, t_{1} ; \vec{p}^{\prime}, t_{0}\right)\right]}{\operatorname{Tr}\left[\Gamma^{\mathrm{unpol}} G^{N N}\left(\vec{p}^{\prime}, t_{2}\right)\right]} \stackrel{\left(t_{1}-t_{0}\right),\left(t_{2}-t_{1}\right) \gg 1 / \Delta E}{\longrightarrow}\langle x\rangle \times t_{2}+\text { const. }
$$

We then extract the slopes in $t_{2}$ and obtain $T_{1}\left(q^{2}\right), T_{2}\left(q^{2}\right)$, $\left[T_{1}+T_{2}\right]\left(q^{2}\right)_{q, g}$, and $\langle x\rangle_{q, g}$ in the DI the same way as is done for the CI.

\section{STOCHASTIC ESTIMATOR AND VARIANCE REDUCTION}

\section{A. Noise estimate of current loop in DI, gauge-field tensor, and unbiased subtraction}

As we mentioned in Sec. III E, we adopt the complex $Z_{2}$ (or $Z_{4}$ ) noise [63] to compute the current loop in DI, because $Z_{N}$ noise has been shown to have the minimum variance $[63,67]$.

As we can see from Eq. (28), the calculation of the gauge-field tensor involves trace over spin indices of the massless overlap Dirac operator [24,54]. Moreover, $\mathcal{T}_{\{4 i\} g}$ involves trace over color indices [see Eq. (5)], and the corresponding three-point function involves a sum over space. This is basically the same as the quark loop calculation. Since we adopt the Zolotarev approximation for the sign function in the overlap operator, it entails an inversion of the Wilson fermion kernel with multishifts [68]. Thus, we again use the complex $Z_{2}$ noise to estimate the trace of Eq. (28) to construct the glue energy-momentum tensor in Eq. (21).

It has been shown that the off-diagonal matrix element contributions to the variance can be reduced by subtracting a judiciously chosen set of traceless $N \times N$ matrices $Q^{(p)}$ [64], which satisfy $\sum_{n=1}^{N} Q_{n, n}^{(p)}=0, p=1, \ldots, P$. Then, the expectation value is unchanged when $M^{-1}$ is substituted with $M^{-1}-\sum_{p=1}^{P} \lambda_{p} Q^{(p)}\left(\lambda_{p}\right.$ is a constant), and yet the variance can be reduced. A natural choice for the set of traceless matrices is the hopping parameter expansion of the inverse of the Wilson fermion matrix, $D_{W}$ [64], and it has been applied to the study of the quark orbital angular momentum [9], the flavor-singlet scalar meson [69], determinant estimate [70], the quark momentum fraction $\langle x\rangle$ [41], and the strangeness electromagnetic form factor [66]. We see a reduction of the errors by more than a factor of 2 with negligible cost. We shall adopt this unbiased subtraction with hopping expansion of the Wilson Dirac fermion to the fourth order.

\section{B. Discrete symmetries and transformations}

Since both the DI and glue operators are stochastically estimated, the signals for the corresponding three-point functions are usually noisy. To improve the signals, we take advantage of discrete symmetries to further reduce the variance from the gauge noise. We will tap parity, $\gamma_{5}$ hermiticity, and charge- $\gamma_{5}$ hermiticity ( $\mathrm{CH}$ transformation) $[41,71,72]$ to filter out the noise contributions that would be zero with infinite statistics. This is the same idea as the unbiased subtraction in Sec. IVA.

\section{Two-point functions and current loop}

Since the three-point functions for DI are constructed by multiplying (or correlating) the nucleon propagator with the current loop on each gauge configuration, we can consider the parity, $\mathrm{CH}$, and $\gamma_{5}$ transformation properties of each of them. In Table I, we show the outcome of parity and $\mathrm{CH}$ transformations on the polarized and unpolarized nucleon propagators. Here, we use the shorthand notation: $f\left(\vec{p}, t ; t_{0} ; U\right)=\operatorname{Tr}\left[\Gamma^{\text {unpol }} G^{N N}\left(\vec{p}, t ; t_{0} ; U\right)\right]$ and $g\left(\vec{p}, t ; t_{0} ; U\right)=$ $\operatorname{Tr}\left[\Gamma^{\mathrm{pol}} G^{N N}\left(\vec{p}, t ; t_{0} ; U\right)\right]$.

Similarly, the outcome of the parity, $\gamma_{5}$, and $\mathrm{CH}$ transformations for the loop of the energy-momentum tensor in Eq. (39) are shown in Table II.

TABLE I. Table showing the outcome of the parity and $\mathrm{CH}$ transformations on unpolarized and polarized nucleon propagators with equal and opposite momenta. $U^{p}$ and $U^{*}$ denote the parity and $C$ transformed gauge links, respectively.

\begin{tabular}{lcc}
\hline \hline Nucleon propagators & Parity & CH transformations \\
\hline$f\left(\vec{p}, t ; t_{0} ; U\right)$ & $f\left(-\vec{p}, t ; t_{0} ; U^{p}\right)$ & {$\left[f\left(-\vec{p}, t ; t_{0} ; U^{*}\right)\right]^{*}$} \\
$f\left(\vec{p}, t ; t_{0} ; U\right)+f\left(-\vec{p}, t ; t_{0} ; U\right)$ & Even & {$\left[f\left(\vec{p}, t ; t_{0} ; U^{*}\right)+f\left(-\vec{p}, t ; t_{0} ; U^{*}\right)\right]^{*}$} \\
$g\left(\vec{p}, t ; t_{0} ; U\right)$ & $g\left(-\vec{p}, t ; t_{0} ; U^{p}\right)$ & $-\left[g\left(-\vec{p}, t ; t_{0} ; U^{*}\right)\right]^{*}$ \\
\hline \hline
\end{tabular}


TABLE II. Table showing the outcome of the parity, $\gamma_{5}$, and $\mathrm{CH}$ transformations on the quark loop for the energy-momentum tensor in Eq. (39).

\begin{tabular}{lccc}
\hline \hline Loop & Parity & $\gamma_{5}$ hermiticity & CH transformations \\
\hline$L\left[t_{1}, \vec{q} ; U\right]$ & $-L\left[t_{1},-\vec{q} ; U^{p}\right]$ & $\frac{+i}{8 a} \sum_{\vec{x}_{1}} e^{i \vec{q} \cdot\left(\vec{x}_{1}-\vec{x}_{0}\right)} \operatorname{Re}\left[L\left[x_{1} ; U\right]\right]$ & $-\left[L\left[t_{1},-\vec{q} ; U^{*}\right]\right]^{*}$ \\
\hline \hline
\end{tabular}

\section{Construction of disconnected three-point functions}

According to the charge conjugation and $\gamma_{5}$ hermiticity $(\mathrm{CH})$ theorem [60], after gauge averaging, the path integral for $\langle\mathcal{O}\rangle$ in $\mathrm{QCD}$ is either real or imaginary (except in the case with chemical potential). Using the transformation properties given in Tables I and II, one can decide on the right combination of real and imaginary components of the nucleon propagator and the loop to satisfy the total parity and $\mathrm{CH}$ transformation properties and the $\gamma_{5}$ hermiticity for the quark loop $[9,41,66]$. In this way, we obtain the unpolarized three-point functions (DI) as

$$
\begin{aligned}
\operatorname{Tr}[ & \left.\Gamma^{\text {unpol }} G^{N \mathcal{T}_{4 i} N}\left(\vec{p}^{\prime}, t_{2} ; \vec{q}, t_{1} ; \vec{p}, t_{0}\right)\right]_{\mathrm{DI}} \\
= & \left(\frac{1}{8 a}\right)\left\langle\left\{\sum_{\vec{x}_{2}} \cos \left(\vec{p}^{\prime} \cdot\left(\vec{x}_{2}-\vec{x}_{0}\right)\right) \operatorname{Re}\left[N^{\mathrm{unpol}}\left[x_{2} ; U\right]\right] \sum_{\vec{x}_{1}} \sin \left(\vec{q} \cdot\left(\vec{x}_{1}-\vec{x}_{0}\right)\right) \operatorname{Re}\left[L\left[x_{1} ; U\right]\right]\right.\right. \\
& \left.\left.-\sum_{\vec{x}_{2}} \sin \left(\vec{p}^{\prime} \cdot\left(\vec{x}_{2}-\vec{x}_{0}\right)\right) \operatorname{Re}\left[N^{\mathrm{unpol}}\left[x_{2} ; U\right]\right] \sum_{\vec{x}_{1}} \cos \left(\vec{q} \cdot\left(\vec{x}_{1}-\vec{x}_{0}\right)\right) \operatorname{Re}\left[L\left[x_{1} ; U\right]\right]\right\}\right\rangle
\end{aligned}
$$

and the polarized three-point functions (DI) as

$$
\begin{aligned}
\operatorname{Tr}[ & \left.\Gamma_{l}^{\mathrm{pol}} G^{N \mathcal{T}_{4 i} N}\left(\vec{p}^{\prime}, t_{2} ; \vec{q}, t_{1} ; \vec{p}, t_{0}\right)\right]_{\mathrm{DI}} \\
= & \left(\frac{i}{8 a}\right)\left\langle\left\{\sum_{\vec{x}_{2}} \cos \left(\vec{p}^{\prime} \cdot\left(\vec{x}_{2}-\vec{x}_{0}\right)\right) \operatorname{Im}\left[N_{l}^{\mathrm{pol}}\left[x_{2} ; U\right]\right] \sum_{\vec{x}_{1}} \sin \left(\vec{q} \cdot\left(\vec{x}_{1}-\vec{x}_{0}\right)\right) \operatorname{Re}\left[L\left[x_{1} ; U\right]\right]\right.\right. \\
& \left.\left.-\sum_{\vec{x}_{2}} \sin \left(\vec{p}^{\prime} \cdot\left(\vec{x}_{2}-\vec{x}_{0}\right)\right) \operatorname{Im}\left[N_{l}^{\mathrm{pol}}\left[x_{2} ; U\right]\right] \sum_{\vec{x}_{1}} \cos \left(\vec{q} \cdot\left(\vec{x}_{1}-\vec{x}_{0}\right)\right) \operatorname{Re}\left[L\left[x_{1} ; U\right]\right]\right\}\right\rangle .
\end{aligned}
$$

\section{CHOICE OF MOMENTA}

The momenta we shall choose for computing the first moment of the momentum fraction carried by quarks for both CI and DI have been discussed in detail in Ref. [41]. For the case of glue, we shall use the same momenta as in the case of DI.

For angular momenta, we have discussed earlier that (see Sec. III F) we need to combine several kinematics into the ratios in Eq. (41) for CI or in Eq. (45) for DI at a particular $q^{2}$ from which one can separate $T_{1}\left(q^{2}\right), T_{2}\left(q^{2}\right)$, and $T_{3}\left(q^{2}\right)$. For this purpose, we first take several momenta to set up the suitable kinematics. Since both the two-point and three-point functions are subject to larger noise with higher momenta, we have limited ourselves to momenta not exceeding 2 (in lattice units). With these momenta under consideration, we can construct four different values of $q^{2}$ for which $\vec{p} \neq \vec{p}^{\prime} \neq \vec{q} \neq 0$. Since the momentum projection is folded in the sequential source at the sink time $t_{2}$ in the CI computation, we have chosen only the cases for which $\vec{p}^{\prime}=$ $(1,0,0)$ in order to reduce the computational cost. In contrast, the computation of the valence quark propagators in DI is separate from the loop computation in each configuration; this means that the momentum in the nucleon two-point functions can be chosen independently of the momentum transfer carried by the costly loop calculation, only constrained by momentum conservation. This allows us to choose all the available momenta at the same computational cost. Similar is the case for the glue contributions.

\section{SEPARATION OF $T_{1}, T_{2}$, AND $T_{3}$}

In this section, we will discuss how to separate $T_{1}, T_{2}$, and $T_{3}$ at a particular value of $q^{2}$ (for details, see the Appendix). Using the available momenta, we obtain several ratios of three-point to two-point functions (both polarized and unpolarized) for all the three directions of the operator, $\mathcal{T}_{4 i}$, at every $q^{2}$. We then average over the ratios with the same coefficients, $a_{i}$ 's, and extract them either by fitting a constant (for CI) or by fitting a slope (for DI and glue). This results in more than three different equations that contain $T_{1}, T_{2}$, and $T_{3}$ with different coefficients $a_{i}$ 's. Though 
these equations are analytically different, numerically they are correlated since they are computed on the same set of configurations. While solving for $T_{1}, T_{2}$, and $T_{3}$, such correlations must be taken into account. Therefore, we construct a covariance matrix, $C$, between these equations for every $q^{2}$. We then construct $\chi^{2}$ as

$$
\begin{aligned}
\chi^{2}= & \sum_{i j}^{N}\left[R_{i}-\left(a_{1, i} T_{1}+a_{2, i} T_{2}+a_{3, i} T_{3}\right)\right] \\
& \times C_{i j}^{-1}\left[R_{j}-\left(a_{1, j} T_{1}+a_{2, j} T_{2}+a_{3, j} T_{3}\right)\right],
\end{aligned}
$$

where $N$ is the number of equations and $R_{i}$ 's are the fitted values of the ratios. Minimizing the $\chi^{2}$ in Eq. (51) with respect to $T_{1}, T_{2}$, and $T_{3}$, we obtain the three equations

$$
\left[\begin{array}{l}
R_{1}^{\prime} \\
R_{2}^{\prime} \\
R_{3}^{\prime}
\end{array}\right]=\left[\begin{array}{lll}
a_{1}^{1} & a_{2}^{1} & a_{3}^{1} \\
a_{1}^{2} & a_{2}^{2} & a_{3}^{2} \\
a_{1}^{3} & a_{2}^{3} & a_{3}^{3}
\end{array}\right]\left[\begin{array}{l}
T_{1} \\
T_{2} \\
T_{3}
\end{array}\right],
$$

where

$a_{k}^{m}=2 a_{m, i} C_{i j}^{-1} a_{k, j}, \quad R_{k}^{\prime}=2 a_{k, i} C_{i j}^{-1} R_{j}, \quad(m, k=1,2,3)$

and the sum over $i, j$ is implicitly implied. Solving the system of equations in Eq. (52), we can separate $T_{1}, T_{2}$, and $T_{3}$ at that $q^{2}$.

\section{NUMERICAL PARAMETERS}

We use 500 gauge configurations on a $16^{3} \times 24$ lattice generated with Wilson action at $\beta=6.0$ in the quenched approximation. They are produced by the pseudoheatbath algorithm with 10,000 sweeps between consecutive configurations. The values of the hopping parameter we have used are $\kappa=0.154,0.155$, and 0.1555 . The critical hopping parameter, $\kappa_{c}=0.1568$, is obtained by a linear extrapolation to the zero pion mass [73]. Using the nucleon mass to set the lattice spacing at $a=0.11 \mathrm{fm}$, the corresponding pion masses are 650(3), 538(4), and 478(4) MeV, and the nucleon masses are 1291(9), 1159(11), and 1093 (13) $\mathrm{MeV}$, respectively. In the present work, we use the periodic boundary condition in the spatial directions. In the temporal direction, the Dirichlet boundary condition is imposed at $t=1$ and $t=24$. This provides a larger time separations than those available with periodic boundary conditions.

The quark loops for DI and overlap operator for glue are computed separately using complex $Z_{2}$ noise vectors [63]. The number of noise vectors we use for DI is 500 on each gauge configuration. Also for the case of quarks, we shall define two $\kappa$ 's for the quark mass: $\kappa_{v}$ for valence quarks and $\kappa_{\text {loop }}$ for quarks in the current loop in the case of DI. For the strange quark currents, we have fixed $\kappa_{\text {loop }}=0.154$, which is close to the strange quark mass as determined from the $\phi$ meson mass, and $\kappa_{v}$ takes the values $0.154,0.155$, and 0.1555 . For up and down quarks, we consider equal masses for valence quarks and quarks in the current loop, i.e., $\kappa_{\text {loop }}=\kappa_{v}=0.154,0.155$, and 0.1555 . The source time for the quark propagators is fixed at $t_{0}=4$. In the case of CI, the sink time is fixed at $t_{2}=16$.

We estimate the gauge-field tensor from the overlap operator stochastically with two complex $Z_{2}$ noise vectors on each configuration, but with dilution in color and spin indices. For the space-time points, we perform a dilution with multiple grids to cover the whole space-time points. The points on the grid are separated by two sites on top of odd/even dilution. Therefore, the taxi-driver distance equals 4 in our case. The reason behind the grid dilution approach is that, unlike the quark loop, the overlap operator is exponentially local with a range of falloff to be about two lattice spacings in the taxi-driver distance.

We use multiple nucleon sources (16 in this work) to increase the statistics in the cases of DI and glue. We correlate all the corresponding two-point functions with the already computed DI and the glue energy-momentum tensor. This has shown to reduce the error significantly $[41,66]$. In the case of CI, we use only one nucleon source.

The error analysis is performed by using the jackknife procedure. The correlations among different quantities are taken into account by constructing the corresponding covariance matrices. To extract various physical quantities, we use correlated least $\chi^{2}$ fits. To determine $T_{1}(0)$ and $T_{2}(0)$, we first separate $T_{1}\left(q^{2}\right), T_{2}\left(q^{2}\right)$, and $T_{3}\left(q^{2}\right)$ at finite $q^{2}$ using the method discussed in Sec. VI for every jackknife sample. $T_{1}(0)$ and $T_{2}(0)$ are then obtained by extrapolating $q^{2}$ to zero with a dipole form. Alternatively, $T_{1}(0)$ can be directly computed from the forward matrix element as discussed in Sec. III F 2. We should point out that we do not take into account the forward matrix value of $T_{1}(0)$ when we perform the $q^{2} \rightarrow 0$ extrapolation for $T_{1}\left(q^{2}\right)$. The values of $T_{1}(0)$ obtained from both the methods are consistent within errors and presented in Sec. VIII. Since $T_{1}(0)$ obtained from forward matrix element is more precise with a smaller error, we shall use it in the following discussion as well as combining with $q^{2}$-extrapolated value of $T_{2}(0)$ to construct $2 J$.

\section{RESULTS AND DISCUSSION}

\section{A. Connected insertions}

We first present our results for the CI. The analyses are a straightforward extension of those in Ref. [41]. In Fig. 2(a), we plot $\left[T_{1}^{u}\left(q^{2}\right)+T_{2}^{u}\left(q^{2}\right)\right]$ and $\left[T_{1}^{d}\left(q^{2}\right)+T_{2}^{d}\left(q^{2}\right)\right]$ as functions of $q^{2}$ for $\kappa=0.1555$, the smallest quark mass, where $T_{1}\left(q^{2}\right)$ and $T_{2}\left(q^{2}\right)$ are obtained by using Eqs. (41) and (52). We also plot $\left[T_{1}+T_{2}\right]^{u}\left(q^{2}\right)$ and $\left[T_{1}+T_{2}\right]^{d}\left(q^{2}\right)$ obtained directly from Eqs. (42) and (43) at slightly different but 


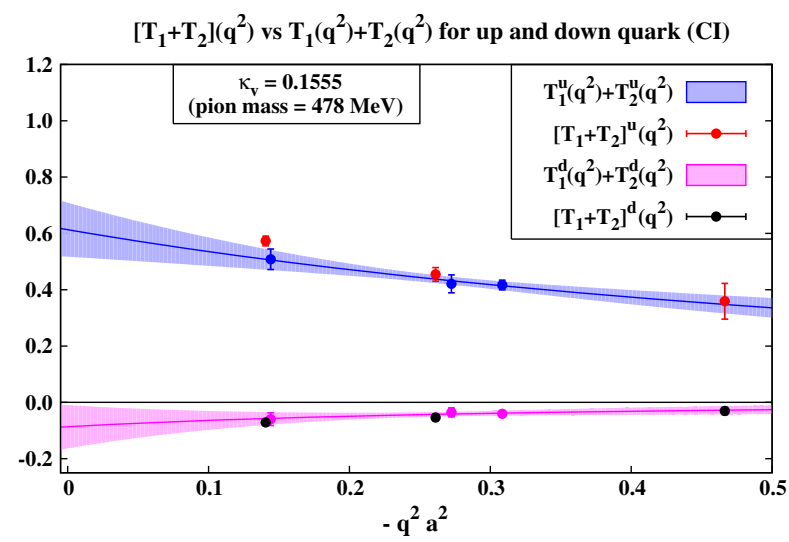

(a)

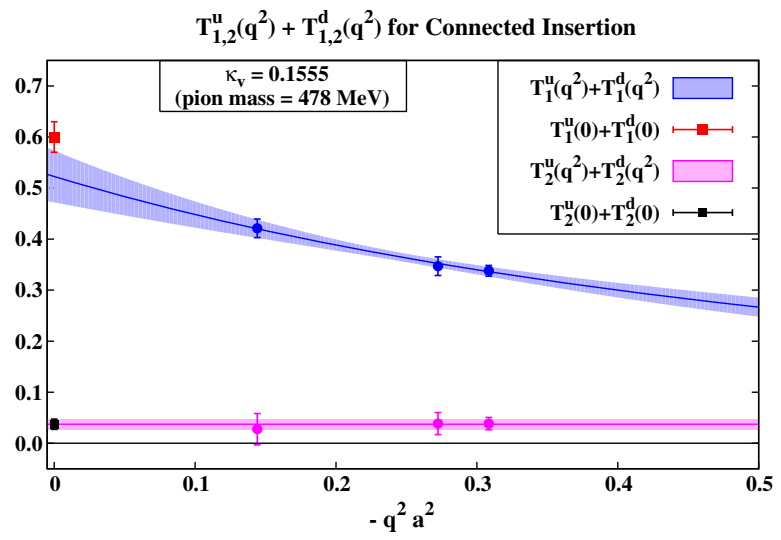

(b)

FIG. 2 (color online). CI plots at $\kappa=0.1555$. (a) The sum of $T_{1}\left(q^{2}\right)$ and $T_{2}\left(q^{2}\right)$, extracted from Eqs. (41) and (52) along with error bands from the dipole fit, is compared to $\left[T_{1}+T_{2}\right]\left(q^{2}\right)$ obtained from Eqs. (42) and (43) at comparable $q^{2}$ values for $u$ and $d$ quarks in the CI. (b) The sum of $u$ and $d$ quark contributions for $T_{1}\left(q^{2}\right)$ and $T_{2}\left(q^{2}\right)$. The red square at $q^{2}=0$ is $\left[T_{1}^{u}(0)+T_{1}^{d}(0)\right]$, which is obtained from forward matrix elements using Eq. (44). The black square at $q^{2}=0$ is $\left[T_{2}^{u}(0)+T_{2}^{d}(0)\right]$, which is obtained from dipole fit. To construct $J^{u+d}(\mathrm{CI})$, we add the values represented by the red and black squares.

comparable $q^{2}$ 's. We see that the latter agrees within $2 \sigma$ of the error band of the former, which is obtained from a dipole fit in $q^{2}$. This is a cross-check of our procedure of extracting $T_{1}\left(q^{2}\right)$ and $T_{2}\left(q^{2}\right)$. We also show $\left[T_{1}^{u}\left(q^{2}\right)+T_{1}^{d}\left(q^{2}\right)\right]$ and $\left[T_{2}^{u}\left(q^{2}\right)+T_{2}^{d}\left(q^{2}\right)\right]$ and their error bands in Fig. 2(b). Also plotted is $\left[T_{1}^{u}(0)+T_{1}^{d}(0)\right]$ from Eq. (44). We see that its error is smaller than that from the separately extrapolated $T_{1}^{u}(0)$ and $T_{1}^{d}(0)$. Thus, we shall use $\left[T_{1}^{u}(0)+T_{1}^{d}(0)\right]$ obtained from Eq. (44) and combine with $\left[T_{2}^{u}(0)+T_{2}^{d}(0)\right]$ obtained from the dipole fit to get the angular momentum $J^{q}$ for the CI. We follow the similar procedure for other $\kappa_{v}$ values.

\section{B. Disconnected insertions}

For the DI, we show one of the ratios in Eq. (45) plotted against the sink time, $t_{2}$, in Fig. 3(a) and the ratio in Eq. (46) similarly plotted in Fig. 3(b) with $\kappa_{v}=\kappa_{\text {loop }}=$ 0.1555 at $q^{2}=0.144$.

We fit the slope from $t_{2}=8$ where the two-point function begins to be dominated by the nucleon to $t_{2}=12$. We plot $\left[T_{1}+T_{2}\right]\left(q^{2}\right)$ so obtained in Fig. 3(c) and compare them to $T_{1}\left(q^{2}\right)+T_{2}\left(q^{2}\right)$ extracted from six combinations of $a_{1} T_{1}\left(q^{2}\right)+a_{2} T_{2}\left(q^{2}\right)+a_{3} T_{3}\left(q^{2}\right)$. We see that they are consistent with each other within errors. The error bands are from the dipole fits of $T_{1}\left(q^{2}\right)$ and $T_{2}\left(q^{2}\right)$. $T_{1}(0)$ (in red squares) is from the forward matrix element, which has smaller error than the $q^{2}$ extrapolated value of $T_{1}(0)$. Thus, in a similar manner as in CI, we shall combine it with the extrapolated $T_{2}(0)$ to obtain the angular momentum $J^{q}$ (DI). We follow the similar procedure for other $\kappa$ values and strange quarks.

Finally, we perform a linear chiral extrapolation of $\kappa_{v}$ to obtain $T_{1}(0)+T_{2}(0)$ for the $u, d$ quarks at the chiral limit.
This is shown in Fig. 3(d). For the strange quark, on the other hand, we fix the loop at $\kappa_{\text {loop }}=0.154$ and then extrapolate the $\kappa_{v}$ to the chiral limit.

\section{Glue}

We perform the similar analysis for the glue momentum and angular momentum. The plots for the glue first moment are shown in Figs. 4(a) and 4(b). For angular momentum, they are plotted in Figs. 5(a)-5(c), and 5(d). The first clear signal for the glue momentum fraction was seen with the overlap operator [54]. Recently, the glue momentum fraction was calculated by using the Feynman-Hellmann theorem [74]. In our current work, clear signals of both the glue momentum and angular momentum fractions have been observed with direct calculation of the glue operators in the nucleon.

In Table III, we list the lattice results on the quark momentum fractions $\langle x\rangle \equiv T_{1}(0)$ for CI $(u$ and $d)$ and DI $(u / d$ and $s$ ) as well as that for glue. We also list the corresponding $T_{2}(0)$ and total angular momenta fraction $2 J=T_{1}(0)+T_{2}(0)$ for each quark flavor and glue. As explained in Sec. VIII A, the $T_{2}(0)$ at $q^{2}=0$ for $\mathrm{CI}(u)$ and $\mathrm{CI}(d)$ are obtained from separate dipole fits in $q^{2}$ as shown in Fig. 2, while the $T_{2}(0)$ for $\mathrm{CI}(u+d)$ is obtained from the dipole fit of the sum of $\mathrm{CI}(u)$ and $\mathrm{CI}(d)$ that leads to a smaller error than that obtained from the separate dipole fits. We note that the $T_{2}(0)$ from the quark and the glue sectors have similar magnitude but with opposite sign that results in cancellation within errors. This is consistent with Eq. (19), which results from momentum and angular momentum conservation. Consequently, the total unrenormalized momentum, $\langle x\rangle^{q}+\langle x\rangle^{g}=0.95(7)$, and angular momentum, $2 J^{q}+2 J^{g}=0.95(9)$, are the 


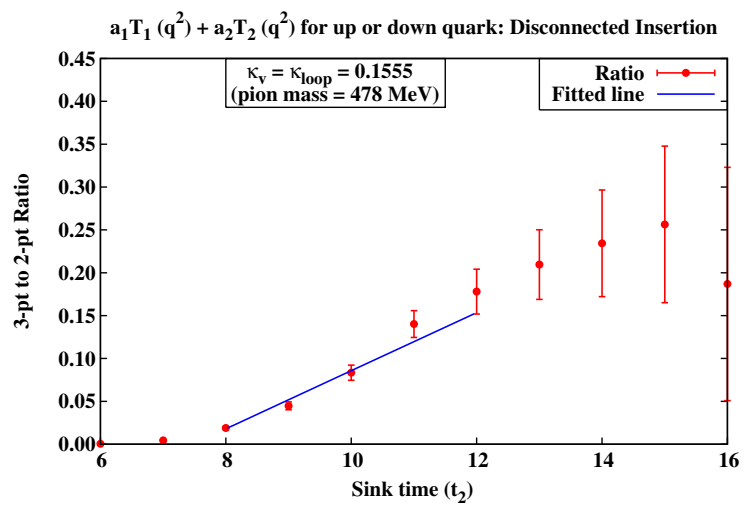

(a)

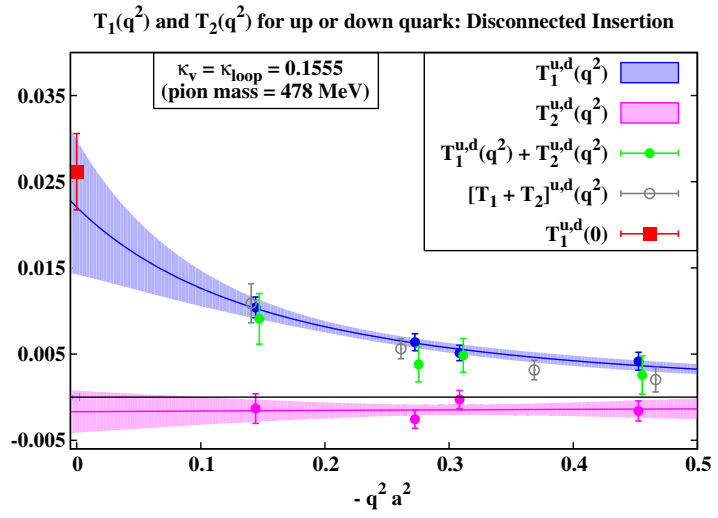

(c)

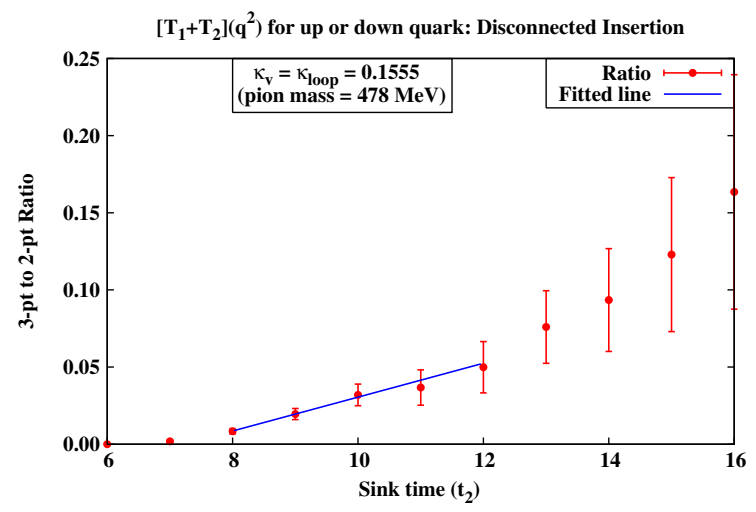

(b)

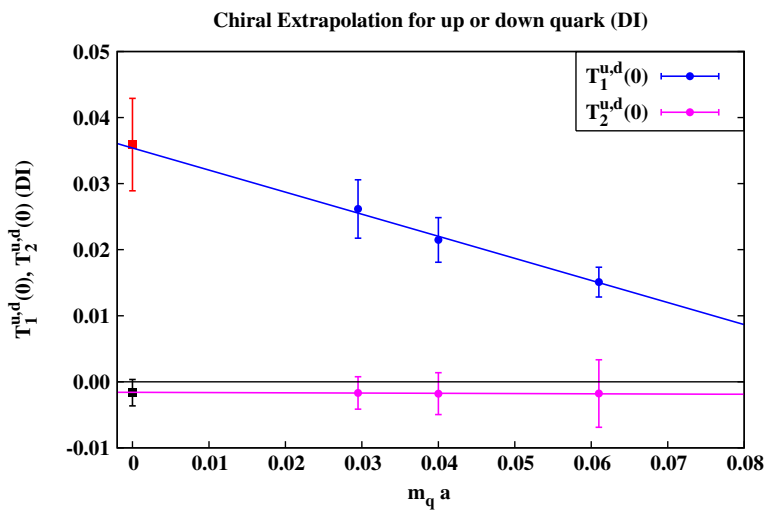

(d)

FIG. 3 (color online). DI plots for $u, d$ at $\kappa_{v}=\kappa_{\text {loop }}=0.1555$. (a) One of the ratios in Eq. (45) plotted against the sink time, $t_{2}$. The term with form factor $T_{3}\left(q^{2}\right)$ does not appear in this particular ratio. The slope is fitted to obtain $\left[a_{1} T_{1}\left(q^{2}\right)+a_{2} T_{2}\left(q^{2}\right)\right]^{u, d}$. (b) The ratio in Eq. (46) plotted against the sink time, $t_{2}$. The slope is fitted to obtain $\left[T_{1}+T_{2}\right]^{u, d}\left(q^{2}\right)$. (c) The sum of separately extracted $T_{1}\left(q^{2}\right)$ and $T_{2}\left(q^{2}\right)$ is compared with $\left[T_{1}+T_{2}\right]\left(q^{2}\right) . T_{1}(0)$ (red square) is from the forward matrix element. To construct $J$, the value represented by the red square is used as $T_{1}(0)$. (d) Chiral extrapolation of $T_{1}(0)$ and $T_{2}(0)$ for the $u / d$ quark. The red and black squares in this figure represent chirally extrapolated values of $T_{1}(0)$ and $T_{2}(0)$, respectively. Please note that they are not renormalized in this figure.

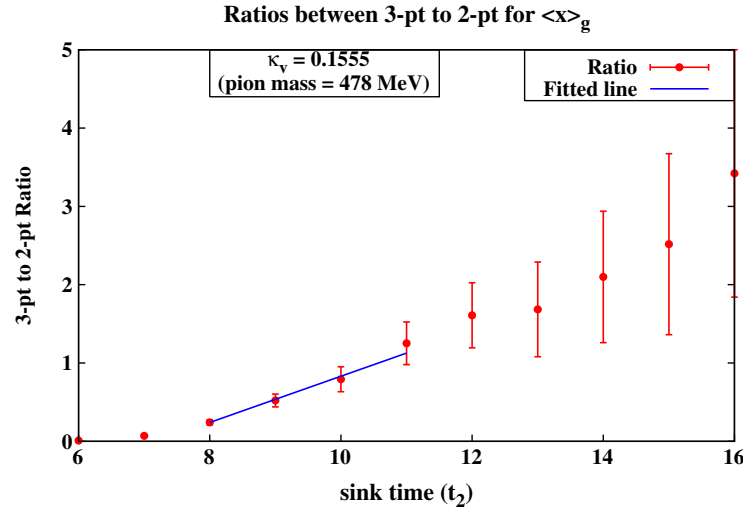

(a)

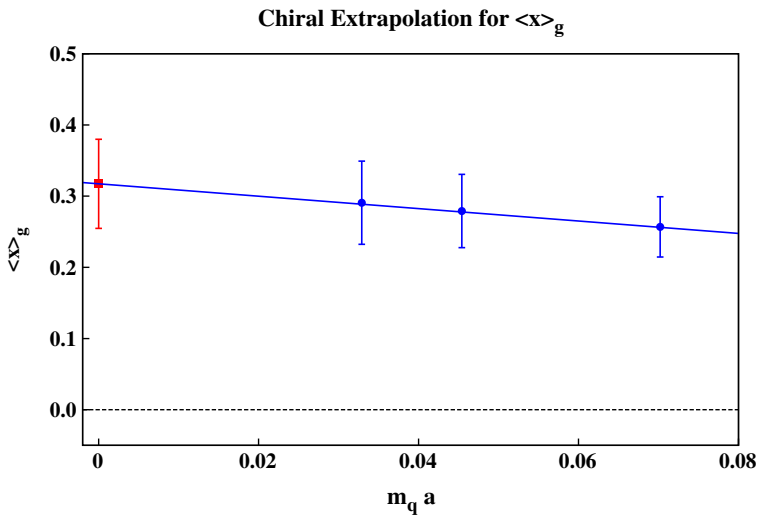

(b)

FIG. 4 (color online). Plots for glue first moment: (a) ratio between three-point and two-point functions obtained by using Eq. (48) at $\kappa_{v}=0.1555$ and (b) chiral extrapolation. 


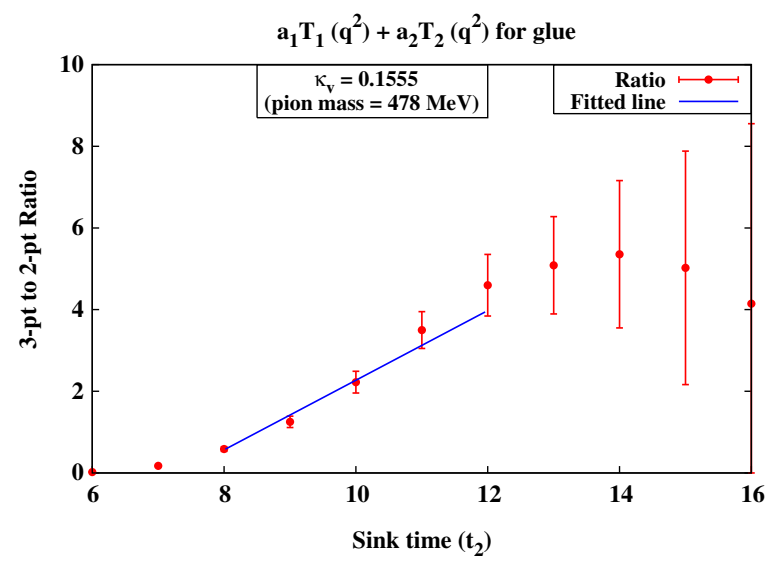

(a)

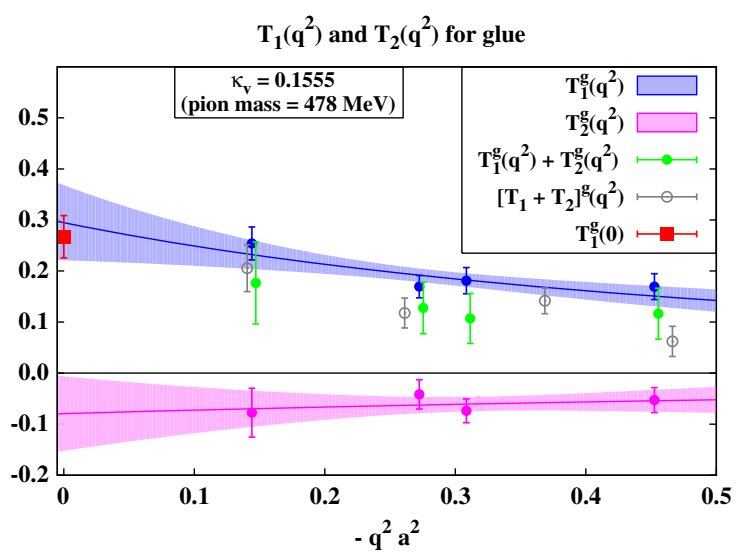

(c)

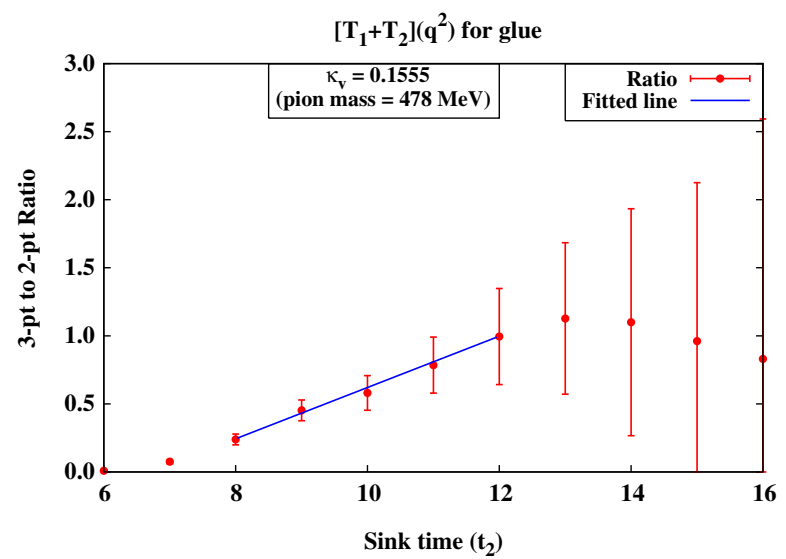

(b)

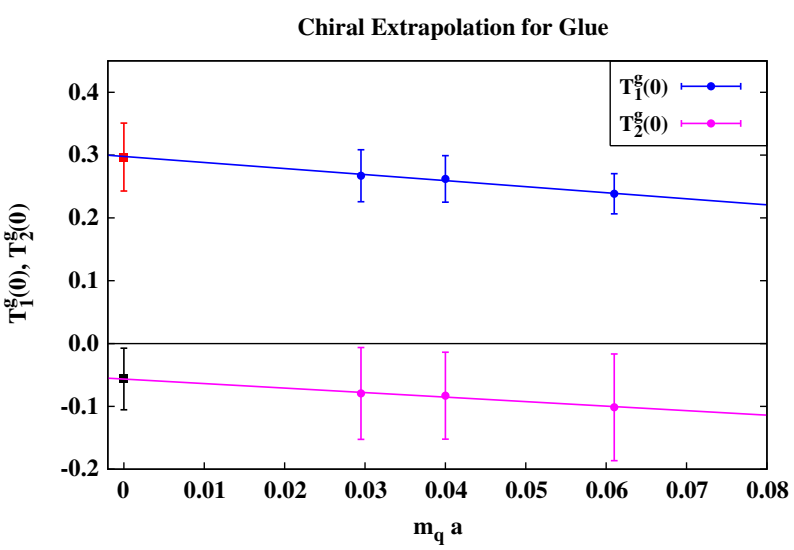

(d)

FIG. 5 (color online). Similar types of plots as in Fig. 3 for the glue at $\kappa_{v}=0.1555$.

same within errors and consistent with and close to unity.

\section{Renormalization}

Before presenting the final results, we discuss renormalization and mixing of quark and glue operators and matching to the $\overline{\mathrm{MS}}$ scheme at a certain scale. The momenta $\langle x\rangle$ and angular momenta $J$ for the quarks and glue are calculated with lattice regularization. To match to the $\overline{\mathrm{MS}}$ scheme at a scale $\mu$ in order to be able to compare with experiments, the renormalized matching and mixing of the momentum fraction (and angular momentum) can be written in the matrix equation

$$
\begin{aligned}
{\left[\begin{array}{c}
\langle x\rangle_{q}^{\overline{\mathrm{MS}}}(\mu, \mathrm{CI}) \\
\langle x\rangle_{q}^{\overline{\mathrm{MS}}}(\mu, \mathrm{DI}) \\
\langle x\rangle_{g}^{\overline{\mathrm{MS}}}(\mu)
\end{array}\right] } & =\left[\begin{array}{ccc}
Z_{q q}\left(a \mu, g_{0}\right) & 0 & 0 \\
0 & Z_{q q}\left(a \mu, g_{0}\right) & Z_{q g}\left(a \mu, g_{0}\right) \\
Z_{g q}\left(a \mu, g_{0}\right) & Z_{g q}\left(a \mu, g_{0}\right) & Z_{g g}\left(a \mu, g_{0}\right)
\end{array}\right] \\
& \times\left[\begin{array}{c}
\langle x\rangle_{q}^{L}(\mathrm{CI}) \\
\langle x\rangle_{q}^{L}(\mathrm{DI}) \\
\langle x\rangle_{g}^{L}
\end{array}\right]
\end{aligned}
$$

where the $\langle x\rangle_{q}^{L}$ and $\langle x\rangle_{g}^{L}$ are lattice matrix elements that satisfy the momentum sum rule and the subscript $q$ refers to the flavor-singlet quark component. The CI part corresponds to the moment of the parton distribution function for the valence and connected-sea (CS) quarks, whereas the DI part is the corresponding moment for the disconnected sea (DS) [59]. The valence, CS, and DS parton degrees of freedom are defined in the path-integral formulation of the hadronic tensor [59], and the separation of CS from DS patrons has been achieved [75] by combining HERMES data on the strangeness distribution [76], the CT10 globally fitted parton distribution functions, and the lattice calculation of the ratio of $\langle x\rangle$ of the strange to that of $u$ (or d) in the DI [54]. It is important to note that valence and CS parton moments do not have contributions from the glue moment. Only the DS patron moment receives contributions from the glue moments through mixing. Since the energy-momentum tensors for the quark and glue are gauge invariant operators, their matrix elements do not mix with those of gauge-variant operators [77].

The quark and glue momentum fractions in the $\overline{\mathrm{MS}}$ scheme sum to unity provided the scheme-dependent renormalization constants, $Z\left(a \mu, g_{0}\right)$ 's, satisfy the constraints $[77,78]$ 
TABLE III. Unrenormalized lattice results of quark and glue momenta and angular momenta.

\begin{tabular}{|c|c|c|c|c|c|c|c|}
\hline & $\mathrm{CI}(u)$ & $\mathrm{CI}(d)$ & $\mathrm{CI}(u+d)$ & $\mathrm{DI}(u / d)$ & $\mathrm{DI}(s)$ & Glue & Total \\
\hline$\langle x\rangle$ & $0.408(38)$ & $0.149(19)$ & $0.558(43)$ & $0.036(7)$ & $0.023(6)$ & $0.298(53)$ & $0.95(7)$ \\
\hline$T_{2}(0)$ & $0.283(107)$ & $-0.217(76)$ & $0.061(20)$ & $-0.002(2)$ & $-0.001(3)$ & $-0.056(49)$ & $0.00(6)$ \\
\hline $2 J$ & $0.691(122)$ & $-0.069(78)$ & $0.620(48)$ & $0.034(7)$ & $0.022(7)$ & $0.242(73)$ & $0.95(9)$ \\
\hline
\end{tabular}

$$
Z_{q q}+Z_{g q}=1, Z_{q g}+Z_{g g}=1,
$$

and the lattice quark and glue momentum fractions are normalized to satisfy the momentum sum rule, i.e.,

$$
\langle x\rangle_{q}^{L}+\langle x\rangle_{g}^{L}=1
$$

where $\langle x\rangle_{q}^{L}=\langle x\rangle_{q}^{L}(\mathrm{CI})+\langle x\rangle_{q}^{L}(\mathrm{DI})$. The sum-rule improved lattice matrix elements in Eq. (56) are defined as

$$
\langle x\rangle_{q, g}^{L}=Z_{q, g}^{L}\langle x\rangle_{q, g}^{L},
$$

where $\langle x\rangle_{q, g}^{L}$ are the unrenormalized matrix elements from the lattice calculation and $Z_{q, g}^{L}$ are the lattice normalization constants that account for lattice systematics.

Since both the momenta and angular momenta are derived from the same energy-momentum tensor operators, both $Z_{q}^{L}$ and $Z_{g}^{L}$ can be determined from the momentum and angular momentum sum rules

$$
\begin{aligned}
Z_{q}^{L}\langle x\rangle_{q}^{L}+Z_{g}^{L}\langle x\rangle_{g}^{L} & =1, \\
Z_{q}^{L} J_{q}^{L}+Z_{g}^{L} J_{g}^{L} & =\frac{1}{2} .
\end{aligned}
$$

Even though the lattice calculated momenta and angular momenta are correlated, the direct fitting of $Z_{q, g}^{L}$ can lead to large errors since the values of $\langle x\rangle_{q}^{L}$ and $2 J_{q}^{L}$ are close, as are those of $\langle x\rangle_{g}^{L}$ and $2 J_{g}^{L}$. The condition number of the $2 \times 2$ matrix of these matrix elements is $\sim 17$. Instead, one can choose to fit $Z_{q, g}^{L}$ from the momentum sum rule in Eq. (58) and

$$
Z_{q}^{L} T_{2, q}^{L}(0)+Z_{g}^{L} T_{2, g}^{L}(0)=0
$$

which leads to a smaller condition number of $\sim 8.6$, but the uncertainties in the lattice normalization factors $Z_{q, g}^{L}$ can still be large.

In view of the fact that the total unrenormalized lattice momentum $\langle x\rangle^{q}+\langle x\rangle^{g}=0.95(7)$ and angular momentum $2 J^{q}+2 J^{g}=0.95(9)$ are the same within errors, we shall simply scale both to unity with $Z_{q}^{L}=Z_{g}^{L}=1.05$ and ignore their errors in this work.

For the renormalization constants, $Z_{q q}, Z_{q g}, Z_{g q}$, and $Z_{g g}$ in Eq. (54), we shall compute them perturbatively. Lattice perturbation calculation has been carried out to match the energy-momentum tensor operators from the lattice to the $\overline{\mathrm{MS}}$ scheme [79]. To one-loop order, they are

$Z_{q q}=1+\frac{g_{0}^{2}}{16 \pi^{2}} C_{F}\left(\frac{8}{3} \log \left(a^{2} \mu^{2}\right)+f_{q q}\right)$,

$Z_{q g}=-\frac{g_{0}^{2}}{16 \pi^{2}}\left(\frac{2}{3} N_{f} \log \left(a^{2} \mu^{2}\right)+f_{q g}\right)$,

$Z_{g q}=-\frac{g_{0}^{2}}{16 \pi^{2}} C_{F}\left(\frac{8}{3} \log \left(a^{2} \mu^{2}\right)+f_{g q}\right)$,

$Z_{g g}=1+\frac{g_{0}^{2}}{16 \pi^{2}}\left(\frac{2}{3} N_{f} \log \left(a^{2} \mu^{2}\right)+f_{g g}\right)$.

For the negative mass parameter $\rho=1.368$ used in the overlap operator, we obtain $f_{q q}=-7.60930$, $f_{g q}=-2.37600, f_{q g}=0.0$, and $f_{g g}=-3.76900$. The details of the calculation are presented in Ref. [79].

We note that if we do not use the sum-rule constraints for the lattice results; i.e., if we set $Z_{q, g}^{L}=1$, we find the total momentum fraction to be 0.92(7) and two times the total angular momentum fraction to be $0.92(9)$ in the $\overline{\mathrm{MS}}$ scheme at $\mu=2 \mathrm{GeV}$ through Eq. (61).

We see that while the scheme- and scale-independent factors associated with the anomalous dimensions $\gamma_{i j}$ together with the unity in the diagonal terms in Eq. (54) satisfy the constraints in Eq. (55), the scheme-dependent finite factors $f_{i j}$ do not. This may be attributed to the artifact in the off-shell calculation of renormalization factors [80]. In the literature, the finite factors $f_{q q}$ and $f_{q g}$ have been calculated to determine $Z_{q q}$ and $Z_{q g}$. On the other hand, $Z_{g q}$ and $Z_{g g}$ are simply defined from the constraints in Eq. (55) $[44,74,81]$ as

$$
Z_{g q}=1-Z_{q q}, \quad Z_{g g}=1-Z_{q g} .
$$

Since we have calculated all the finite factors $f_{i j}$, we shall consider the average of the procedure such as the one in Eq. (62) and replace $f_{i j}$ in Eq. (61) with $\tilde{f}_{i j}$ given by

$\tilde{f}_{q q}=\tilde{f}_{g q}=\frac{1}{2}\left(f_{q q}+f_{g q}\right), \quad \tilde{f}_{q g}=\tilde{f}_{g g}=\frac{1}{2}\left(f_{q g}+f_{g g}\right)$ 
so that the constraints in Eq. (55) are satisfied. Although this procedure has an ambiguity, this systematic is expected to make negligible contributions to the final momentum and angular momentum fractions in the $\overline{\mathrm{MS}}$ scheme. Since the prefactor $g_{0}^{2} /\left(16 \pi^{2}\right)=6.33 \times 10^{-3}$ is small, the effects in the finite factors in the renormalization constants are much smaller than unity. We find that the corresponding differences in the quark and glue momentum fractions due to the finite factors that are obtained by using Eqs. (62) and (63) are less than 1\%, which is much smaller than the statistical errors of the physical quantities we calculate.

Since our inverse lattice spacing is determined to be $1 / a=1.74 \mathrm{GeV}$ from the nucleon mass [9], $\log \left(a^{2} \mu^{2}\right)=$ 0.279 is small because $1 / a$ is close to the scale $\mu \simeq 2 \mathrm{GeV}$. Moreover, the factor $g_{0}^{2} /\left(16 \pi^{2}\right)=6.33 \times 10^{-3}$ is also small. As a result, the diagonal renormalization coefficients $Z_{q q}=0.9641$ and $Z_{q q}=0.9881$ (for the quenched case with $N_{f}=0$ ) are close to unity, and the off-diagonal mixing coefficients $Z_{g q}=0.0359$ and $Z_{q g}=0.0119$ are close to zero. We see from Eq. (54) that there are only subpercent changes from the lattice results to those in the $\overline{\mathrm{MS}}$ scheme at $\mu \simeq 2 \mathrm{GeV}$. We report our results in the $\overline{\mathrm{MS}}$ scheme at $\mu=2 \mathrm{GeV}$.

\section{E. Discussion}

In Table IV, we list the renormalized quark momentum fractions $\langle x\rangle \equiv T_{1}(0)$ for CI $(u$ and $d)$ and DI $(u / d$ and $s)$ as well as that of glue. We also list the corresponding $T_{2}(0)$ and total angular momenta fraction $2 J=T_{1}(0)+T_{2}(0)$ for each quark flavor and glue. These values are obtained at $\mu=2 \mathrm{GeV}$ in $\overline{\mathrm{MS}}$ scheme as explained in Sec. VIII D. To obtain results for different flavor, we note that $\langle x\rangle_{q}^{L}(\mathrm{CI})$ is the linear sum of those of $u$ and $d$ in the CI, and $\langle x\rangle_{q}^{L}(\mathrm{DI})$ is the linear sum of those of $u, d$, and $s$ in the DI. Thus, in practice, Eq. (54) is extended to the bases of the direct product of flavor and CI and DI plus the glue, and the renormalization constants in Eq. (61) modified in such a way that $N_{F}$ is replaced with unity and $f_{q g}$ is replaced with $1 / N_{F}$. The exception to this change is $Z_{g g}$, where the $N_{F}$ factor is zero for the present quenched calculation.

We see from Table IV that the strange momentum fraction $\langle x\rangle_{s}=0.024(6)$ is in the range of uncertainty of $\langle x\rangle_{s}$ from the CTEQ fitting of the parton distribution function from experiments, which is $0.018<\langle x\rangle_{s}<$ 0.040 [82]. The glue momentum fraction of $0.334(55)$ is smaller than, say, the CTEQ4M fit of 0.42 at $\mu=1.6 \mathrm{GeV}$ [83], but only by $1.5 \sigma$. The smallness of our value of $\langle x\rangle_{g}$ in comparison to the experiment could be in part due to the fact that ours is a quenched calculation. We expect the glue momentum fraction to be larger than the present result when dynamical configurations with a light fermion are used in the calculation.

From Figs. 2(b) and 5(c) and Table IV, we find that $\left[T_{2}^{u}(0)+T_{2}^{d}(0)\right](\mathrm{CI})$ is positive and $T_{2}^{g}(0)$ is negative so that the total sum including the small $\left[T_{2}^{u}(0)+T_{2}^{d}(0)+\right.$ $T_{2}^{s}(0)$ ] (DI) can be naturally constrained to be zero [see Eq. (19)] with the normalization constants $Z_{q}^{L}=1.05$ and $Z_{g}^{L}=1.05$ close to unity. In analogy to $F_{2}(0)$, the anomalous magnetic moment of the nucleon, $T_{2}(0)$, is termed as the anomalous gravitomagnetic moment and has been shown to vanish for composite systems by Brodsky et al. [38]. As we explained in Sec. II, the vanishing of the total $T_{2}(0)$ is the consequence of momentum and angular momentum conservation.

The flavor-singlet $g_{A}^{0}$, which is the quark spin contribution to the nucleon, has been calculated before on the same lattice [2]. We can subtract it from the total quark angular momentum fraction $2 J$ to obtain the orbital angular momentum fraction $2 L$ for the quarks. As we see in Table IV, the orbital angular momentum fractions $2 L$ for the $u$ and $d$ quarks in the CI have different signs, and they add up to zero, i.e., 0.01(10). This is the same pattern seen with dynamical fermions configurations with light quarks [11-15]. The large $2 L$ for the $u / d$ and $s$ quarks in the DI is due to the fact that $g_{A}^{0}$ in the DI is large and negative, i.e., -0.12 (1) for each of the three flavors. Altogether, the quark orbital angular momentum constitutes a fraction of 0.47 (13) of the nucleon spin. The majority of it comes from the DI. The quark spin fraction of the nucleon spin is $0.25(12)$, and the glue angular momentum contributes a fraction of $0.28(8)$. We show all the different contributions to the momentum, angular momenta, and orbital angular momenta in Figs. 6(a)-6(c). The left panels show the combinations of $u$ and $d$ contributions from CI and DI separately, while the right panels show the contributions

TABLE IV. Renormalized values in the $\overline{\mathrm{MS}}$ scheme at $\mu=2 \mathrm{GeV}$.

\begin{tabular}{|c|c|c|c|c|c|c|}
\hline & $\mathrm{CI}(u)$ & $\mathrm{CI}(d)$ & $\mathrm{CI}(u+d)$ & $\mathrm{DI}(u / d)$ & $\mathrm{DI}(s)$ & Glue \\
\hline$\overline{\langle x\rangle}$ & $0.413(38)$ & $0.150(19)$ & $0.565(43)$ & $0.038(7)$ & $0.024(6)$ & $0.334(55)$ \\
\hline$T_{2}(0)$ & $0.286(108)$ & $-0.220(77)$ & $0.062(21)$ & $-0.002(2)$ & $-0.001(3)$ & $-0.056(51)$ \\
\hline $2 J$ & $0.700(123)$ & $-0.069(79)$ & $0.628(49)$ & $0.036(7)$ & $0.023(7)$ & $0.278(75)$ \\
\hline$g_{A}$ & $0.91(11)$ & $-0.30(12)$ & $0.62(9)$ & $-0.12(1)$ & $-0.12(1)$ & - \\
\hline $2 L$ & $-0.21(16)$ & $0.23(15)$ & $0.01(10)$ & $0.16(1)$ & $0.14(1)$ & - \\
\hline
\end{tabular}




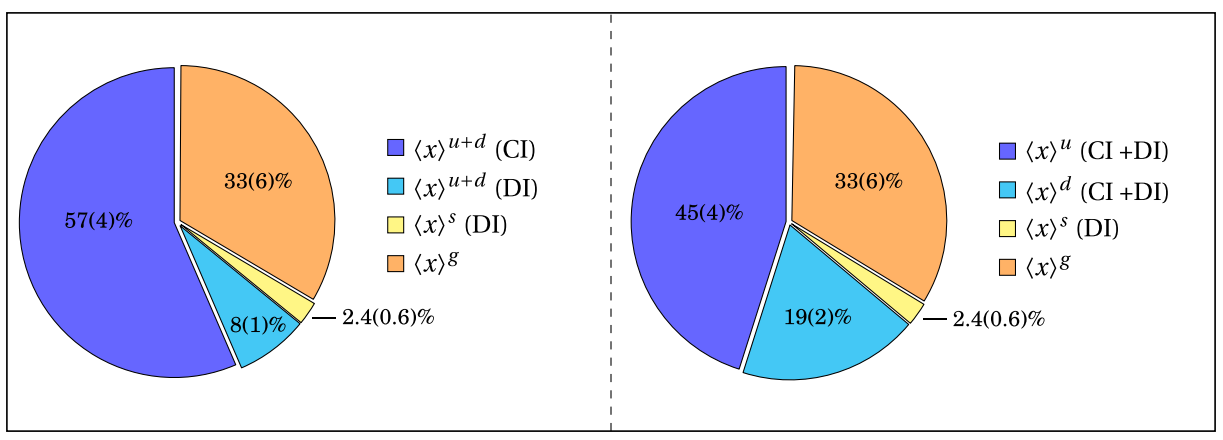

(a)

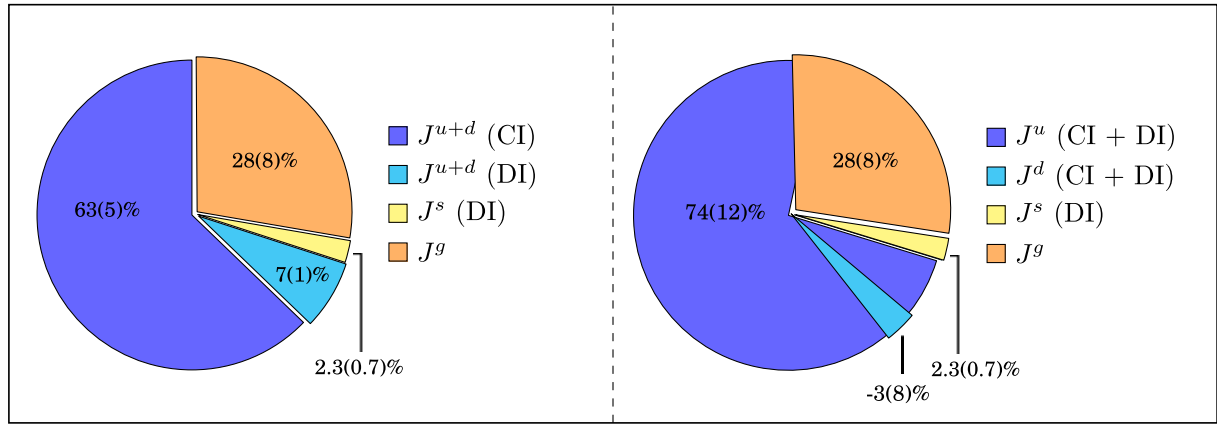

(b)

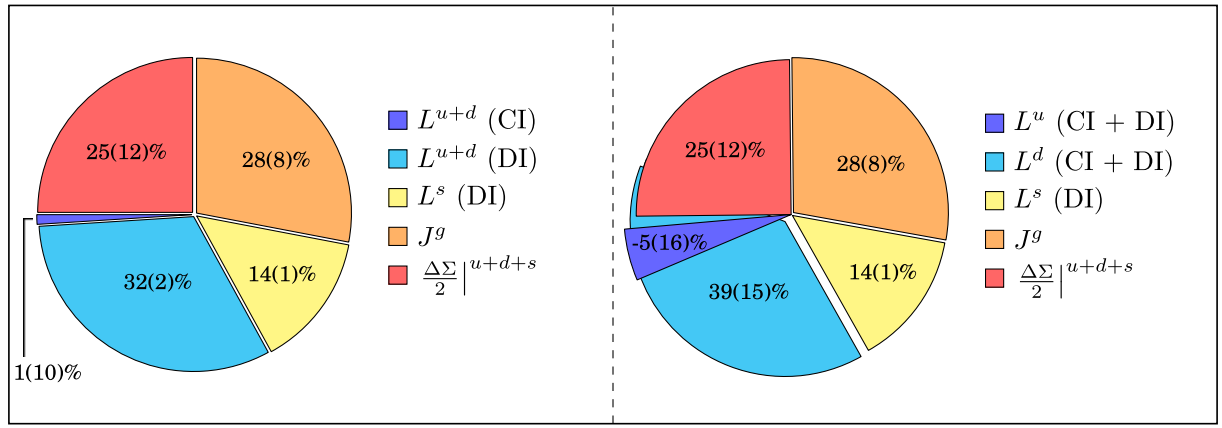

(c)

FIG. 6 (color online). Pie charts for the quark and gluon contributions to the (a) momentum fraction, (b) angular momenta, and (c) orbital angular momenta. The left panels show the quark contributions separately for CI and DI, and the right panels show the quark contributions for each flavor with CI and DI summed together for $u$ and $d$ quarks.

from the $u$ and $d$ quarks (with both CI and DI combined together).

We note from Table IV that the orbital angular momenta contribution from each quark flavor is strongly dependent on the corresponding quark spin, particularly in the case of DI. As opposed to earlier calculations [2-4], the recent lattice calculations with light dynamical fermions [5-7] have obtained smaller quark spin contributions from DI. However, preliminary study [8] of the anomalous Ward identity with light valence overlap fermions on $2+1$-flavor dynamical domain wall fermion sea configurations suggests that the DI contributions are not small, though this study has larger error bars. More detailed dynamical fermion calculations with controlled statistical and systematic errors are needed to settle this issue.

We should point out that a small $\Delta u+\Delta d+\Delta s$ from the DI does not explain the small quark spin $g_{A}^{0}=(\Delta u+$ $\Delta d)(\mathrm{CI})+(\Delta u+\Delta d+\Delta s)(\mathrm{DI}) \sim 0.25$ from the global fitting of DIS [1], in view of the fact that most of the lattice calculation of $(\Delta u+\Delta d)(\mathrm{CI})$ is $\sim 0.6$, which is much larger than 0.25 . On the other hand, one could imagine that $(\Delta u+\Delta d)(\mathrm{CI})$ may turn out to be smaller than 0.6 when the quark mass is close to the physical one in future lattice calculations, such as in Ref. [14], where $(\Delta u+\Delta d)(\mathrm{CI})$ is found to be much smaller than 0.6 when the chiral extrapolation of the lattice results is carried out. 
However, this will not explain the octet $g_{A}^{8}=$ $(\Delta u+\Delta d)(\mathrm{CI})+(\Delta u+\Delta d-2 \Delta s)(\mathrm{DI})$. When both the $\mathrm{CI}$ and DI are small, the calculated $g_{A}^{8}$ will be smaller than the experimental value of $g_{A}^{8}=0.579(25)$ [84]. Thus, it is difficult to explain simultaneously $g_{A}^{0}$ and $g_{A}^{8}$ with a small $(\Delta u+\Delta d+\Delta s)$ in DI. To clarify this issue, a full QCD simulation for $g_{A}^{0}$ and $g_{A}^{8}$ (both CI and DI) around the physical point by taking into account the $S U(3)$ breaking effect is necessary.

In the constituent quark model, the proton spin comes entirely from the quark spin. On the other hand, in the skyrmion, the total proton spin is from the collective rotational motion of the pion field [85]. What we find in the present calculation seems to suggest that the QCD picture, aside from the glue contribution, is somewhere in between these two models. Following Wilson's renormalization group approach to effective theories, it is suggested [86] that the effective theory for baryons between the scale of $4 \pi f_{\pi}$ and $\sim 300 \mathrm{MeV}$ may be a chiral quark model with renormalized couplings and renormalized meson, quark, and gluon fields that preserve chiral symmetry. Models like the little bag model with the skyrmion outside the MIT bag [87], the cloudy bag model [88], and quark chiral soliton model [89] could possibly delineate the pattern of division among the components of the proton spin with large quark orbital angular momentum contribution.

\section{SUMMARY}

In summary, we have carried out a complete calculation of the quark and glue momentum and angular momentum in the nucleon for the first time on a quenched $16^{3} \times 24$ lattice with three quark masses. The calculation includes both the CI and DI of the three-point functions for the quark energy-momentum tensor. We have used complex $Z_{2}$ noise to estimate the quark loops in the DI and the gauge-field tensor from the overlap operator in the glue energymomentum tensor. We find that reasonable signals can be obtained for the glue operator constructed from the overlap Dirac operator. After chiral extrapolation, the momentum and angular momentum sum rules are used to normalize the quark and glue momentum and angular momentum fractions on the lattice. The renormalization and mixing of the quark and glue energy-momentum operators are obtained through one-loop perturbation, and the final results are reported in the $\overline{\mathrm{MS}}$ scheme at $2 \mathrm{GeV}$. The renormalized momentum fractions for the quarks are $0.565(43)$ for the CI and $0.100(15)$ for the DI. The glue momentum fraction is $0.334(55)$. We have demonstrated that the vanishing anomalous gravitomagnetic moment [see Eq. (19)] is a consequence of momentum and angular momentum conservation.

After subtracting the quark spin $\left(g_{A}^{0}\right)$ from a previous calculation on the same lattice [2] from the angular momentum $2 J$, we obtain the orbital angular fraction $2 L$. In the $\mathrm{CI}$, we find that the $u$ quark contribution is negative, while the $d$ quark contribution is positive. The sum is 0.01(10), which is small. This behavior is the same as observed in dynamical calculation with light quarks [11-15]. The majority of the quark orbital angular momentum turns out to come from the DI because the quark spin from the DI is large and negative for each of the three flavors. In the end, we find the quark orbital angular momentum, the quark spin, and glue angular momentum fractions of the nucleon spin are $0.47(13), 0.25(12)$, and $0.28(8)$, respectively.

Finally, this work should be extended to dynamical fermion calculations with light quarks and continuum and large volume limits to control the systematic errors of lattice QCD. We are in the process of carrying out the same calculation with the valence overlap fermion on $2+1$-flavor dynamical domain wall fermion sea configurations to remove the systematic errors due to the quenched approximation.

\section{ACKNOWLEDGMENTS}

This work is partially supported by U.S. DOE Grant No. DE-FG05-84ER40154 and the Center for Computational Sciences of the University of Kentucky. The work of M. Deka is partially supported by the Institute of Mathematical Sciences, India. The work of T. Doi is supported in part by MEXT Grant-in-Aid for Young Scientists (B) (Grant No. 24740146). We would like to thank Igor V. Anikin, Ying Chen, and Oleg V. Teryaev for useful discussions and comments.

\section{APPENDIX: SOLVING SYSTEM OF KINEMATICAL EQUATIONS}

In this section, we will discuss how to solve a system of kinematical equations to extract $T_{1}, T_{2}$, and $T_{3}$. As mentioned in Sec. III F, we need to combine several kinematics into the ratios in Eq. (41) for CI or in Eq. (45) for DI at a particular $q^{2}$ in order to separate $T_{1}\left(q^{2}\right), T_{2}\left(q^{2}\right)$, and $T_{3}\left(q^{2}\right)$. Using the available momenta, we obtain several ratios (for both polarized and unpolarized nucleons) for all the three directions of the operator, $\mathcal{T}_{4 i}$, at every $q^{2}$. From these ratios, one can set up kinematical equations to solve for $T_{1}, T_{2}$, and $T_{3}$. For simplicity, we will consider the CI only as we have considered $\vec{p}^{\prime}=(1,0,0)$ in this case in order to reduce computational cost. The procedure for DI will be similar except that we will have more available momenta.

If we consider the lowest $q^{2}(=0.1460$ for $\kappa=0.154)$ and $\vec{p}^{\prime}=(1,0,0)$, we obtain the following five different equations as 


$$
\begin{aligned}
& \frac{1}{4}\left[R_{41}^{\mathrm{unpol}}(0,1,0)+R_{41}^{\mathrm{unpol}}(0,-1,0)+R_{41}^{\mathrm{unpol}}(0,0,1)+R_{41}^{\mathrm{unpol}}(0,0,-1)\right] \\
& =\frac{1}{4} \frac{1}{\sqrt{E_{p^{\prime}}\left(E_{p^{\prime}}+m\right) E_{p}\left(E_{p}+m\right)}} \\
& \times\left[T_{1}\left(q^{2}\right)\left\{p_{1}^{\prime}\left(E_{p^{\prime}}+E_{p}\right)\left(3 E_{p^{\prime}}+E_{p}+4 m\right)\right\}\right. \\
& \left.+\frac{1}{2 m} T_{2}\left(q^{2}\right)\left\{p_{1}^{\prime}\left(E_{p^{\prime}}-E_{p}\right)^{2}\left(E_{p^{\prime}}+E_{p}\right)-p_{1}^{\prime} q_{2}^{2}\left(3 E_{p^{\prime}}+E_{p}+2 m\right)\right\}\right], \\
& \frac{1}{4}\left[R_{42}^{\mathrm{unpol}}(0,1,0)-R_{42}^{\mathrm{unpol}}(0,-1,0)+R_{43}^{\mathrm{unpol}}(0,0,1)-R_{43}^{\mathrm{unpol}}(0,0,-1)\right] \\
& =\frac{1}{4} \frac{1}{\sqrt{E_{p^{\prime}}\left(E_{p^{\prime}}+m\right) E_{p}\left(E_{p}+m\right)}} \\
& \times\left[T_{1}\left(q^{2}\right)\left\{\left(-2 q_{2}\right)\left(E_{p^{\prime}}+m\right)\left(E_{p^{\prime}}+E_{p}\right)\right\}+\frac{1}{2 m} T_{2}\left(q^{2}\right)\left\{\left(-q_{2}\right)\left(E_{p^{\prime}}+m\right)\left(E_{p^{\prime}}^{2}+E_{p}^{2}-q_{2}^{2}\right)\right\}\right. \\
& \left.+\frac{2}{m} T_{3}\left(q^{2}\right)\left\{q_{2}\left(E_{p^{\prime}}-E_{p}\right)\left(E_{p^{\prime}}+m\right)\left(E_{p}-E_{p^{\prime}}+2 m\right)\right\}\right] \\
& \frac{1}{2}\left[R_{41}^{\mathrm{pol}}(0,1,0)-R_{41}^{\mathrm{pol}}(0,-1,0)\right]=\frac{1}{4} \frac{1}{\sqrt{E_{p^{\prime}}\left(E_{p^{\prime}}+m\right) E_{p}\left(E_{p}+m\right)}}\left[T_{1}\left(q^{2}\right)\left\{\left(-q_{2}\right)\left(\left(E_{p^{\prime}}+m\right)\left(E_{p^{\prime}}+E_{p}\right)+2 p_{1}{ }^{\prime 2}\right)\right\}\right. \\
& \left.+\frac{1}{2 m} T_{2}\left(q^{2}\right)\left\{\left(-q_{2}\right)\left(E_{p^{\prime}}+m\right)^{2}\left(E_{p^{\prime}}+E_{p}\right)-p_{1}^{\prime} q_{2}^{2}\left(3 E_{p^{\prime}}+3 E_{p}+4 m\right)\right\}\right] \text {, } \\
& \frac{1}{2}\left[R_{42}^{\mathrm{pol}}(0,1,0)-R_{42}^{\mathrm{pol}}(0,-1,0)\right]=\frac{1}{4} \frac{1}{\sqrt{E_{p^{\prime}}\left(E_{p^{\prime}}+m\right) E_{p}\left(E_{p}+m\right)}} \\
& \times\left[T_{1}\left(q^{2}\right)\left\{\left(-p_{1}^{\prime}\right)\left(E_{p^{\prime}}^{2}-E_{p}^{2}-q_{2}^{2}\right)\right\}+\frac{1}{2 m} T_{2}\left(q^{2}\right)\left\{\left(-p_{1}^{\prime}\right)\left(E_{p^{\prime}}+E_{p}+2 m\right)\left(E_{p^{\prime}}^{2}-E_{p}^{2}-q_{2}^{2}\right)\right\}\right. \\
& \left.+\frac{2}{m} T_{3}\left(q^{2}\right)\left\{p_{1}^{\prime} q_{2}^{2}\left(E_{p^{\prime}}-E_{p}\right)\right\}\right] \text {, } \\
& \frac{1}{2}\left[R_{42}^{\mathrm{pol}}(0,0,1)-R_{42}^{\mathrm{pol}}(0,0,-1)\right]=\frac{1}{4} \frac{1}{\sqrt{E_{p^{\prime}}\left(E_{p^{\prime}}+m\right) E_{p}\left(E_{p}+m\right)}} \\
& \times\left[T_{1}\left(q^{2}\right)\left\{\left(-p_{1}^{\prime}\right)\left(E_{p^{\prime}}^{2}-E_{p}^{2}\right)\right\}+\frac{1}{2 m} T_{2}\left(q^{2}\right)\left\{\left(-p_{1}^{\prime}\right)\left(E_{p^{\prime}}^{2}-E_{p}^{2}\right)\left(E_{p^{\prime}}+E_{p}+2 m\right)\right.\right. \\
& \left.\left.+\left(-p_{1}^{\prime}\right)\left(E_{p^{\prime}}+E_{p}\right) q_{3}^{2}\right\}\right]
\end{aligned}
$$

where $R$ 's are the ratios in Eq. (41); e.g., the notation $R_{41}^{\text {unpol }}(0,1,0)$ signifies the ratio for the unpolarized threepoint functions corresponding to the $\mathcal{T}_{41}$ operator with a momentum transfer of $\vec{q}=(0,1,0)$.

For convenience, we shall write the Eqs. (A1), (A2), (A3), (A4), and (A5) in the following manner:

$$
\begin{aligned}
& R_{1}=a_{1,1} T_{1}\left(q^{2}\right)+a_{2,1} T_{2}\left(q^{2}\right)+a_{3,1} T_{3}\left(q^{2}\right), \\
& R_{2}=a_{1,2} T_{1}\left(q^{2}\right)+a_{2,2} T_{2}\left(q^{2}\right)+a_{3,2} T_{3}\left(q^{2}\right),
\end{aligned}
$$

$$
\begin{aligned}
& R_{3}=a_{1,3} T_{1}\left(q^{2}\right)+a_{2,3} T_{2}\left(q^{2}\right)+a_{3,3} T_{3}\left(q^{2}\right), \\
& R_{4}=a_{1,4} T_{1}\left(q^{2}\right)+a_{2,4} T_{2}\left(q^{2}\right)+a_{3,4} T_{3}\left(q^{2}\right), \\
& R_{5}=a_{1,5} T_{1}\left(q^{2}\right)+a_{2,5} T_{2}\left(q^{2}\right)+a_{3,5} T_{3}\left(q^{2}\right) .
\end{aligned}
$$

Here, $a_{i, j}$ 's are the constant coefficients of $T_{1}\left(q^{2}\right), T_{2}\left(q^{2}\right)$, and $T_{3}\left(q^{2}\right)$, which include the factor, $\frac{1}{4} \frac{1}{\sqrt{E_{p^{\prime}}\left(E_{p^{\prime}}+m\right) E_{p}\left(E_{p}+m\right)}}$. However, the Eqs. (A6), (A7), (A8), (A9), and (A10), 
though different, are numerically correlated since they are computed on the same set of configurations. Such correlations are taken into account by constructing a covariance matrix, $C$, between these equations. This allows us to define the corresponding $\chi^{2}$ as

$$
\begin{aligned}
\chi^{2}= & \sum_{i j}^{N}\left[R_{i}-\left(a_{1, i} T_{1}+a_{2, i} T_{2}+a_{3, i} T_{3}\right)\right] \\
& \times C_{i j}^{-1}\left[R_{j}-\left(a_{1, j} T_{1}+a_{2, j} T_{2}+a_{3, j} T_{3}\right)\right],
\end{aligned}
$$

where $N$ is the number of equations that is equal to 5 in this case. We then solve for $T_{1}, T_{2}$, and $T_{3}$ by imposing the following minimization conditions on the $\chi^{2}$ obtained from Eq. (A11):

$$
\frac{\partial \chi^{2}}{\partial T_{1}}=0, \quad \frac{\partial \chi^{2}}{\partial T_{2}}=0, \quad \frac{\partial \chi^{2}}{\partial T_{3}}=0 .
$$

This results in the three equations

$$
\begin{aligned}
& R_{1}^{\prime}=a_{1}^{1} T_{1}+a_{2}^{1} T_{2}+a_{3}^{1} T_{3}, \\
& R_{2}^{\prime}=a_{1}^{2} T_{1}+a_{2}^{2} T_{2}+a_{3}^{2} T_{3}, \\
& R_{3}^{\prime}=a_{1}^{3} T_{1}+a_{2}^{3} T_{2}+a_{3}^{3} T_{3},
\end{aligned}
$$

where

$a_{k}^{m}=2 a_{m, i} C_{i j}^{-1} a_{k, j}, \quad R_{k}^{\prime}=2 a_{k, i} C_{i j}^{-1} R_{j}, \quad(m, k=1,2,3)$,

and the sum over $i, j$ is implied according to Einstein's summation rule. Solving Eqs. (A13), (A14) and (A15), we can separate $T_{1}, T_{2}$ and $T_{3}$ at that $q^{2}$.
[1] D. de Florian, R. Sassot, M. Stratmann, and W. Vogelsang, Phys. Rev. D 80, 034030 (2009).

[2] S. J. Dong, J.-F. Lagae, and K. F. Liu, Phys. Rev. Lett. 75, 2096 (1995).

[3] M. Fukugita, Y. Kuramashi, M. Okawa, and A. Ukawa, Phys. Rev. Lett. 75, 2092 (1995).

[4] S. Güsken, P. Ueberholz, J. Viehoff, N. Eicker, T. Lippert, K. Schilling, A. Spitz, and T. Struckmann (TXL Collaboration), Phys. Rev. D 59, 114502 (1999).

[5] G. S. Bali et al. (QCDSF Collaboration), Phys. Rev. Lett. 108, 222001 (2012).

[6] A. Abdel-Rehim, C. Alexandrou, M. Constantinou, V. Drach, K. Hadjiyiannakou, K. Jansen, G. Koutsou, and A. Vaquero, Phys. Rev. D 89, 034501 (2014).

[7] R. Babich, R. C. Brower, M. A. Clark, G. T. Fleming, J. C. Osborn, C. Rebbi, and D. Schaich, Phys. Rev. D 85, 054510 (2012).

[8] M. Gong and K. F. Liu, Lattice 2013, the 31st International Symposium on Lattice Field Theory, Mainz, Germany, 2013 (unpublished).

[9] N. Mathur, S. J. Dong, K. F. Liu, L. Mankiewicz, and N. C. Mukhopadhyay, Phys. Rev. D 62, 114504 (2000).

[10] M. Göckeler, R. Horsley, D. Pleiter, P. Rakow, A. Schäfer, G. Schierholz, and W. Schroers (QCDSF Collaboration), Phys. Rev. Lett. 92, 042002 (2004).

[11] Ph. Hägler, J. Negele, D. Renner, W. Schroers, Th. Lippert, and K. Schilling (LHPC and SESAM Collaborations), Phys. Rev. D 68, 034505 (2003).

[12] J. D. Bratt et al. (LHPC Collaboration), Phys. Rev. D 82, 094502 (2010).

[13] D. Brommel et al. (QCDSF-UKQCD Collaboration), Proc. Sci., LATTICE 2007 (2007) 158.
[14] C. Alexandrou, M. Constantinou, S. Dinter, V. Drach, K. Jansen, C. Kallidonis, and G. Koutsou, Phys. Rev. D 88, 014509 (2013).

[15] C. Alexandrou, J. Carbonell, M. Constantinou, P. A. Harraud, P. Guichon, K. Jansen, C. Kallidonis, T. Korzec, and M. Papinutto, Phys. Rev. D 83, 114513 (2011).

[16] C. Adolph et al. (COMPASS Collaboration), Phys. Rev. D 87, 052018 (2013); Phys. Lett. B 718, 922 (2013).

[17] P. Djawotho (STAR Collaboration), J. Phys. Conf. Ser. 295, 012061 (2011).

[18] A. Airapetian et al. (HERMES Collaboration), J. High Energy Phys. 08 (2010) 130.

[19] M. Stolarski (COMPASS Collaboration), Nucl. Phys. B, Proc. Suppl. 207-208, 53 (2010).

[20] A. Adare et al. (PHENIX Collaboration), Phys. Rev. D 79, 012003 (2009).

[21] E. C. Aschenauer et al., arXiv:1304.0079.

[22] M. G. Alekseev et al. (COMPASS Collaboration), Phys. Lett. B 690, 466 (2010); 693, 227 (2010).

[23] S. J. Brodsky and S. Gardner, Phys. Lett. B 643, 22 (2006).

[24] K. F. Liu, A. Alexandru, and I. Horvath, Phys. Lett. B 659, 773 (2008).

[25] A. Alexandru, I. Horvath, and K.-F. Liu, Phys. Rev. D 78, 085002 (2008).

[26] See, for example, S. Weinberg, Gravitation and Cosmology (Wiley, New York, 1972).

[27] R. Jackiw, Current Algebra and Anomalies (World Scientific, Singapore, 1985), p. 537.

[28] R. L. Jaffe and A. Manohar, Nucl. Phys. B337, 509 (1990).

[29] X.-D. Ji, Phys. Rev. Lett. 78, 610 (1997).

[30] X.-S. Chen, X.-F. Lu, W.-M. Sun, F. Wang, and T. Goldman, Phys. Rev. Lett. 100, 232002 (2008).

[31] M. Wakamatsu, Phys. Rev. D 81, 114010 (2010). 
[32] Y. Hatta, Phys. Rev. D 84, 041701 (2011).

[33] X. Ji, J.-H. Zhang, and Y. Zhao, Phys. Rev. Lett. 111, 112002 (2013).

[34] E. Leader and C. Lorce, arXiv:1309.4235.

[35] W. Wilcox, Phys. Rev. D 66, 017502 (2002).

[36] O. V. Teryaev, arXiv:hep-ph/9904376.

[37] I. Y. Kobzarev and L. B. Okun, Zh. Eksp. Teor. Fiz. 43, 1904 (1962) [Sov. Phys. JETP 16, 1343 (1963)].

[38] S. J. Brodsky, D. S. Hwang, B.-Q. Ma, and I. Schmidt, Nucl. Phys. B593, 311 (2001).

[39] I. Montvay and G. Münster, Quantum Fields on A Lattice (Cambridge University Press, Cambridge, England, 1997).

[40] C. Best, M. Göckeler, R. Horsley, E.-M. Ilgenfritz, H. Perlt, P. Rakow, A. Schäfer, G. Schierholz, A. Schiller, and S. Schramm, Phys. Rev. D 56, 2743 (1997).

[41] M. Deka, T. Streuer, T. Doi, S. J. Dong, T. Draper, K. F. Liu, N. Mathur, and A. W. Thomas, Phys. Rev. D 79, 094502 (2009).

[42] A. S. Kronfeld and D. M. Photiadis, Phys. Rev. D 31, 2939 (1985).

[43] M. Göckeler, R. Horsley, E.-M. Ilgenfritz, H. Oelrich, H. Perlt, P. E. L. Rakow, G. Schierholz, A. Schiller, and P. Stephenson, Nucl. Phys. B, Proc. Suppl. 53, 324 (1997).

[44] H. B. Meyer and J. W. Negele, Phys. Rev. D 77, 037501 (2008).

[45] P. Hernández, K. Jansen, and M. Luscher, Nucl. Phys. B552, 363 (1999).

[46] T. Draper et al., Proc. Sci., LAT 2005 (2006) 120.

[47] T. Draper et al., arXiv:hep-lat/0609034.

[48] I. Horvath, S. J. Dong, T. Draper, F. X. Lee, K. F. Liu, N. Mathur, H. B. Thacker, and J. B. Zhang, Phys. Rev. D 68, 114505 (2003).

[49] I. Horváth, A. Alexandru, J. B. Zhang, Y. Chen, S. J. Dong, T. Draper, K. F. Liu, N. Mathur, S. Tamhankar, and H. B. Thacker, Phys. Lett. B 617, 49 (2005).

[50] Y. Kikukawa and A. Yamada, Phys. Lett. B 448, 265 (1999).

[51] D. H. Adams, Ann. Phys. (Berlin) 296, 131 (2002).

[52] K. Fujikawa, Nucl. Phys. B546, 480 (1999).

[53] H. Suzuki, Prog. Theor. Phys. 102, 141 (1999).

[54] T. Doi et al. ( $\chi \mathrm{QCD}$ Collaboration), Proc. Sci., LATTICE 2008 (2008) 163.

[55] B. L. Ioffe, Nucl. Phys. B188, 317 (1981); 191, 591(E) (1981).

[56] T. Draper, R. M. Woloshyn, and K.-F. Liu, Phys. Lett. B 234, 121 (1990).

[57] K. F. Liu and S. J. Dong, Phys. Rev. Lett. 72, 1790 (1994).

[58] K. F. Liu, S. J. Dong, T. Draper, D. Leinweber, J. H. Sloan, W. Wilcox, and R. M. Woloshyn, Phys. Rev. D 59, 112001 (1999).

[59] K. F. Liu, Phys. Rev. D 62, 074501 (2000).

[60] C. W. Bernard, Gauge Theory on a Lattice, edited by C. Zachos et al. (Argonne National Laboratory, Argonne, IL, 1984), p. 85.

[61] T. Draper, Report No. UMI-84-28507.

[62] C. W. Bernard, T. Draper, G. Hockney, A. M. Rushton, and A. Soni, Phys. Rev. Lett. 55, 2770 (1985).
[63] S. J. Dong and K. F. Liu, Phys. Lett. B 328, 130 (1994).

[64] C. Thron, S. J. Dong, K. F. Liu, and H. P. Ying, Phys. Rev. D 57, 1642 (1998).

[65] L. Maiani, G. Martinelli, M. L. Paciello, and B. Taglienti, Nucl. Phys. B293, 420 (1987).

[66] T. Doi, M. Deka, S.-J. Dong, T. Draper, K. F. Liu, D. Mankame, N. Mathur, and T. Streuer, Phys. Rev. D80, 094503 (2009).

[67] S. Bernardson, P. McCarty, and C. Thron, Comput. Phys. Commun. 78, 256 (1994).

[68] Y. Chen, S. J. Dong, T. Draper, I. Horvath, F. X. Lee, K. F. Liu, N. Mathur, and J. B. Zhang, Phys. Rev. D 70, 034502 (2004).

[69] C. McNeile and C. Michael (UKQCD Collaboration), Phys. Rev. D 63, 114503 (2001).

[70] A. Alexandru, A. Li, and K.-F. Liu, Proc. Sci., LAT 2007 (2007) 167.

[71] C. W. Bernard, Lectures at TASI '89, Boulder, CO, 1989.

[72] T. Draper, R. M. Woloshyn, W. Wilcox, and K. F. Liu, Nucl. Phys. B318, 319 (1989).

[73] S. J. Dong, J. F. Lagae, and K. F. Liu, Phys. Rev. D 54, 5496 (1996).

[74] R. Horsley, R. Millo, Y. Nakamura, H. Perlt, D. Pleiter, P. E. L. Rakow, G. Schierholz, A. Schiller, F. Winter, and J. M. Zanotti (QCDSF and UKQCD Collaborations), Phys. Lett. B 714, 312 (2012).

[75] K.-F. Liu, W.-C. Chang, H.-Y. Cheng, and J.-C. Peng, Phys. Rev. Lett. 109, 252002 (2012).

[76] A. Airapetian et al. (HERMES Collaboration), Phys. Lett. B 666, 446 (2008).

[77] S. D. Joglekar and B. W. Lee, Ann. Phys. (Berlin) 97, 160 (1976).

[78] X.-D. Ji, Phys. Rev. D 52, 271 (1995).

[79] M. Glatzmaier and K. F. Liu, arXiv:1403.7211.

[80] J. C. Collins and R. J. Scalise, Phys. Rev. D 50, 4117 (1994).

[81] C. Alexandrou, V. Drach, K. Hadjiyiannakou, K. Jansen, B. Kostrzewa, and C. Wiese, Proc. Sci., LATTICE 2013 (2013) 289.

[82] H. L. Lai, P. Nadolsky, J. Pumplin, D. Stump, W. K. Tung, and C.-P. Yuan, J. High Energy Phys. 04 (2007) 089.

[83] H. L. Lai, J. Huston, S. Kuhlmann, F. I. Olness, J. F. Owens, D. E. Soper, W. K. Tung, and H. Weerts, Phys. Rev. D 55, 1280 (1997).

[84] F. E. Close and R. G. Roberts, Phys. Lett. B 316, 165 (1993).

[85] G. S. Adkins, C. R. Nappi, and E. Witten, Nucl. Phys. B228, 552 (1983).

[86] K. F. Liu, S. J. Dong, T. Draper, J. H. Sloan, W. Wilcox, and R. M. Woloshyn, Phys. Rev. D 61, 118502 (2000).

[87] G. E. Brown and M. Rho, Phys. Lett. 82B, 177 (1979).

[88] A. W. Thomas, S. Theberge, and G. A. Miller, Phys. Rev. D 24, 216 (1981).

[89] M. Wakamatsu and Y. Nakakoji, Phys. Rev. D 74, 054006 (2006). 